\title{
RAB39B-mediated trafficking of the GluA2-AMPAR subunit controls dendritic spine maturation and intellectual disability-related behaviour
}

\author{
Maria Lidia Mignogna ${ }^{1}$ - Stefano Musardo $\mathbb{1}^{2}$ - Giulia Ranieri ${ }^{1}$. Susanna Gelmini ${ }^{1}$ • Pedro Espinosa $\mathbb{1}^{2}$. \\ Paolo Marra ${ }^{3}$ - Sara Belloli $\mathbb{1}^{4,5} \cdot$ Valentina Murtaj $\mathbb{1}^{5,6} \cdot$ Rosa Maria Moresco $\mathbb{1}^{5,7}$. Camilla Bellone $\mathbb{(}^{2}$. \\ Patrizia D'Adamo (iD) ${ }^{1}$
}

Received: 14 May 2020 / Revised: 19 April 2021 / Accepted: 4 May 2021 / Published online: 25 May 2021

(c) The Author(s) 2021. This article is published with open access

\begin{abstract}
Mutations in the $R A B 39 B$ gene cause X-linked intellectual disability (XLID), comorbid with autism spectrum disorders or early Parkinson's disease. One of the functions of the neuronal small GTPase RAB39B is to drive GluA2/GluA3 $\alpha$-amino-3hydroxy-5-methyl-4-isoxazolepropionic acid receptor (AMPAR) maturation and trafficking, determining AMPAR subunit composition at glutamatergic postsynaptic neuronal terminals. Taking advantage of the Rab39b knockout murine model, we show that a lack of RAB39B affects neuronal dendritic spine refinement, prompting a more $\mathrm{Ca}^{2+}$-permeable and excitable synaptic network, which correlates with an immature spine arrangement and behavioural and cognitive alterations in adult mice. The persistence of immature circuits is triggered by increased hypermobility of the spine, which is restored by the $\mathrm{Ca}^{2+}$-permeable AMPAR antagonist NASPM. Together, these data confirm that RAB39B controls AMPAR trafficking, which in turn plays a pivotal role in neuronal dendritic spine remodelling and that targeting $\mathrm{Ca}^{2+}$-permeable AMPARs may highlight future pharmaceutical interventions for $R A B 39 B$-associated disease conditions.
\end{abstract}

Supplementary information The online version contains supplementary material available at https://doi.org/10.1038/s41380021-01155-5.

Patrizia D'Adamo

dadamo.patrizia@hsr.it

1 Neuroscience Division, Unit of molecular genetics of intellectual disability, IRCCS San Raffaele Scientific Institute, Milan, Italy

2 Department of Basic Neurosciences, University of Geneva, Geneva, Switzerland

3 Preclinical Imaging Facility, Experimental Imaging Center (EIC), IRCCS San Raffaele Scientific Institute, Milan, Italy

4 Institute of Bioimaging and Physiology, CNR, Segrate (MI), Italy

5 Experimental Imaging Center (EIC), IRCCS San Raffaele Scientific Institute, Milan, Italy

6 PhD Program in Neuroscience, University of Milano - Bicocca, Monza, Italy

7 Medicine and Surgery Department, University of Milano Bicocca, Monza (MB), Italy

\section{Introduction}

Genetic alterations, either copy number gain, deletions or point mutations, of the $\mathrm{X}$-linked $R A B 39 B$ locus are associated with complex neuropathological clinical features, ranging from $\mathrm{X}$-linked intellectual disability (XLID) in comorbidity with autism spectrum disorders, macrocephaly and seizures (OMIM: 300774) [1-4] or with early Parkinson's disease (OMIM: 311510) [5-11]. RAB39B is a neuronal RAB GTPase that controls intracellular vesicular trafficking by switching from an active GTP-bound state to an inactive GDP-bound state [12]. We previously showed that RAB39B, in tandem with PICK1, drives GluA2/ GluA3 $\alpha$-amino-3-hydroxy-5-methyl-4-isoxazolepropionic acid receptor (AMPAR) trafficking throughout the secretory pathway to achieve AMPAR subunit insertion at the neuronal glutamatergic postsynaptic terminal. The downregulation of RAB39B in primary hippocampal neurons alters GluA2/GluA3 AMPAR trafficking from the endoplasmic reticulum (ER) to the Golgi apparatus, affecting AMPAR subunit composition as a final step and leading to increased surface expression of GluA2-lacking $\mathrm{Ca}^{2+}$ permeable AMPARs [13]. 
The subunit composition of AMPARs is highly dynamic and is modified during development, in cases of plasticity and by disease. The increase in surface expression of GluA2lacking $\mathrm{Ca}^{2+}$-permeable AMPAR composition is mostly observed after plasticity-inducing neuronal activity [14, 15] or in young animals. Pathological conditions have been associated with immature synapses and cognitive impairment [16].

$R A B 39 B$, similar to many other XLID genes, plays a role in a common cellular pathway that controls glutamatergic synapses during development and network establishment $[17,18]$. One of the most important cellular processes during brain development is spine formation. This event occurs during early postnatal development and is followed by a pruning phase during adolescence when redundant synaptic connections are removed and remodelled to create a mature state [19-21]. During the adult phase, a constant equilibrium between dendritic spine formation and elimination persists, leading to spine maintenance [22, 23]. However, the molecular mechanism underlying spine development and maturation is not totally defined.

Here, by using a Rab39b knockout (KO) murine model, we confirmed that the complete absence of RAB39B leads to increased surface expression of GluA2-lacking $\mathrm{Ca}^{2+}$-permeable AMPARs, which results in an immature spine state caused by unsuccessful neuronal dendritic spine refinement associated with cognitive deficits. Moreover, the persistence of immature spines and aberrant circuits could be triggered by an increase in the hyperdynamic features of spines, often observed at the early developmental neuronal stage.

Last, the re-expression of RAB39B in neurons or the reestablishment of AMPAR surface functionality by using the $\mathrm{Ca}^{2+}$-permeable AMPAR antagonist $N$-[3-[[4-[(3-aminopropyl)amino]butyl]amino]propyl]-1-naphthaleneacetamide-trihydrochloride (NASPM), but not GluA2 per se, causes the morphology and dynamic rate of Rab39b KO spines to revert.

Together, these results highlight a new role for AMPAR trafficking via RAB39B in spine remodelling, explaining how $R A B 39 B$ loss-of-function mutations lead to the pathogenesis of XLID. We propose a new target suitable for future therapeutic interventions.

\section{Methods}

\section{Animals}

Experiments were performed in accordance with animal protocols approved by the "Institutional Animal Care and Use Committee (IACUC)" (IRCCS San Raffaele Scientific Institute, Milan, Italy) and by the Italian National Ministry of Health (IACUC ID 652, 653, 984), following the guidelines established by the European Community Council Directive D.L. 26/2014 on the use of animals in research (86/609/EEC). We minimised animal suffering, and we used only the number of animals necessary to produce reliable results. Animals were maintained on a $12 \mathrm{~h}$ light/ darkness cycle, and an inverted cycle was employed in behavioural studies. Food pellets and water were available ad libitum unless stated otherwise.

\section{Generation of Rab39b knockout mice}

The murine $R a b 39 b$ gene mapped to the mouse $\mathrm{X}$ chromosome in XA7.3 occupying a region of 6187 base pairs (bp) (NC_000086.7). It is composed of two exons of 215 and $427 \mathrm{bp}$ spaced out by one $2785 \mathrm{bp}$ intron. The $5^{\prime}$ - and $3^{\prime}$ - untranslated regions (UTRs) flanking the two exons were $224 \mathrm{bp}$ and $2536 \mathrm{bp}$, respectively.

$R a b 39 b^{-1 Y}$ mice (Rab39b KnockOut, KO) were generated by injecting three gRNAs (Sigma-Aldrich; \#MM0000573711; \#MM0000573712; \#MM0000573713) targeting different DNA sequences in the 1 st Rab39b exon, together with Cas 9 mRNA in $\mathrm{C} 57 \mathrm{Bl} / 6 \mathrm{~N}$ murine zygotes [24]. We obtained 15/76 pups carrying different insertion/ deletion (indel) mutations, and we selected heterozygote females carrying a deletion of $14 \mathrm{bp}$ or an insertion of 10 $\mathrm{bp}$, giving a frame shift and a premature stop codon after 84 and 92 amino acids, respectively, from guide \#MM0000573713. Possible off-targets were excluded by CCTop [25]. Heterozygous females were crossed with C57Bl/6N wild-type (WT) males (Charles River, Italy) to monitor the transmission of the mutant allele in the expected Mendelian segregation ratio of an $\mathrm{X}$ chromosome gene in N1 generation. Genotypes were determined by sequencing the portion of DNA surrounding the mutation (MyGATC from Eurofins Genomics; 1385-primer: 5'-TGTTTGTCA CCCTGGCAGCATCG-3') after PCR amplification with New_5'ARM_Sal_For (5'-GGTTGTCGACCAGGCCAGT GATGTTCTCGCGG-3') and 1385 primers. Generations up to N6 were obtained by backcrossing Rab39b heterozygote females carrying a 14 bp deletion or a $10 \mathrm{bp}$ insertion with C57Bl/6N WT males to establish murine lines. For all the experiments, except for those depicted in Fig. 1b, c, Rab39b KO 14-bp male mice and their WT littermates were used.

Because $R A B 39 B$ is an $\mathrm{X}$-linked gene and because $R A B 39 B$-related XLID disease is inherited as a recessive trait and is more common in male patients, we focused all experiments on male mouse littermates. For body weight, Rab39b WT and KO littermate mice were weighed every 7 days from 7 to 91 days of life $(\mathrm{P})$. The brain, testes, kidney, liver, spleen, heart, lung, stomach of P20 and P90 Rab39b WT and KO littermate mice were weighed, and coordinates of brains were measured as described in [26]. 
a
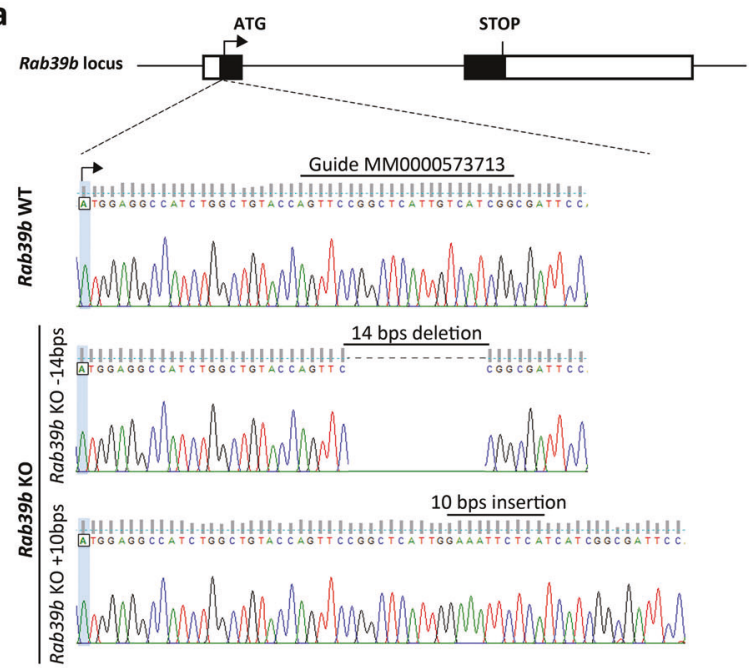

d

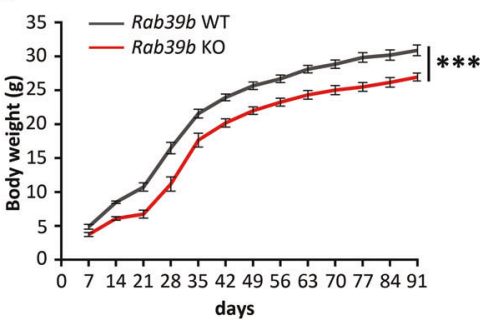

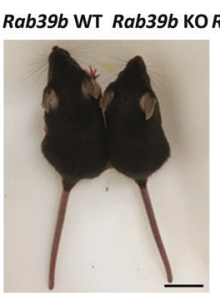

b

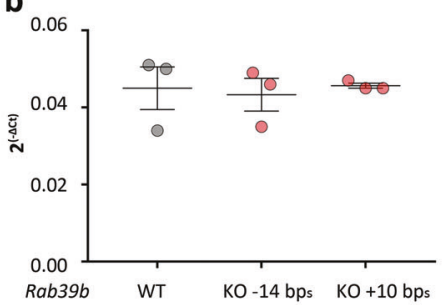

C

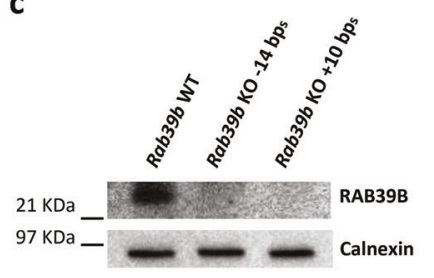

f

P90
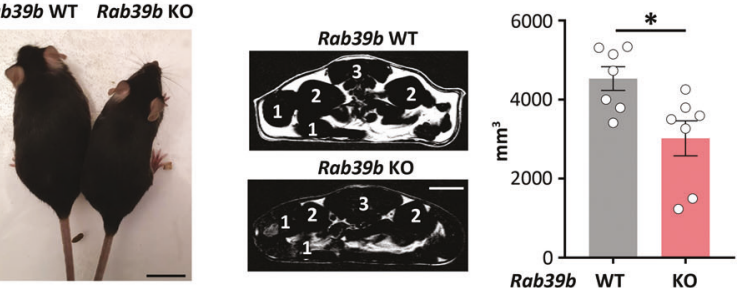

g
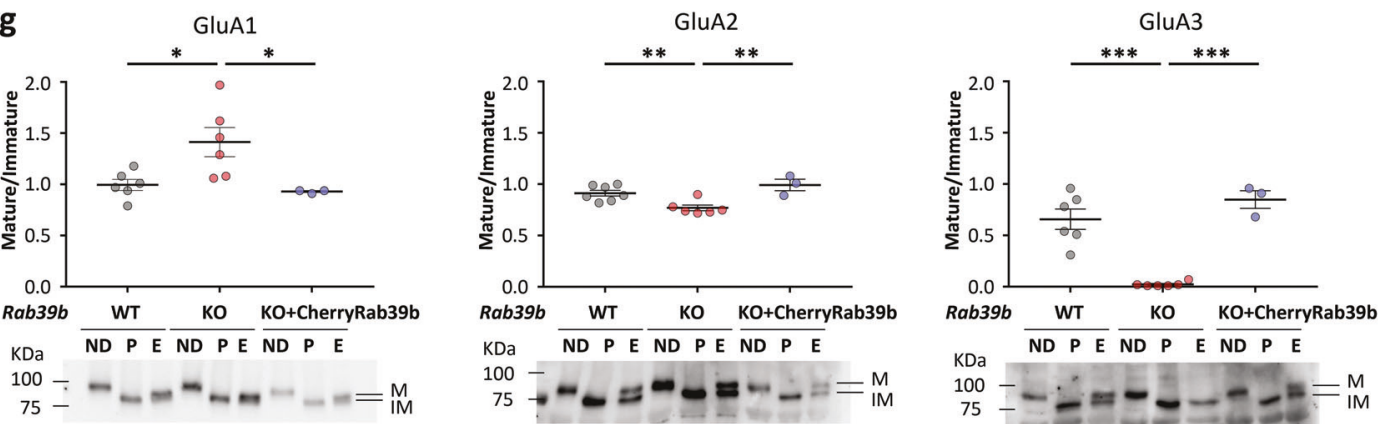

h
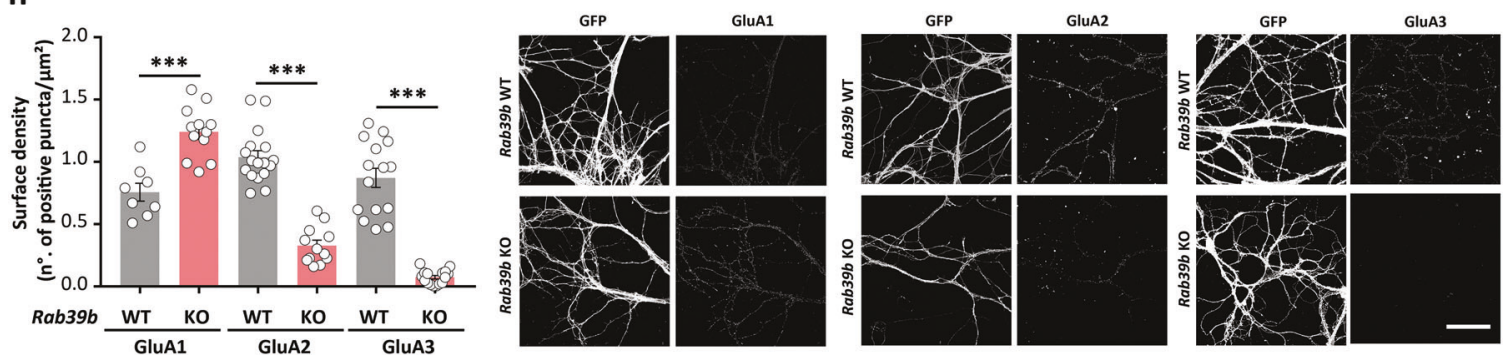

\section{RNA isolation and qRT-PCR}

Total RNA from hippocampal tissues was isolated with TRIzol reagent (Invitrogen, \#15596018) according to the manufacturer's instructions. $1 \mu \mathrm{g}$ of total RNA was reverse transcribed with M-MLV (Invitrogen, \#28025-013) following the manufacturer's instructions to produce cDNA.
Real-time PCR was performed with SYBR Green Universal Mix (Roche, \#04707516001) on a Light Cycler 480 (Roche Diagnostics). Data quantification was performed via the comparative threshold cycle $(\mathrm{Ct})$ method with formula $2^{\text {-(inputCt-controlCt) }}$, assuming that the efficiency was $2 . R a b 39 b$ was amplified with mR39bF (5'-GGATACAGCGGGT CAAGAGAGG-3') and mR39bR (5'-GTTGGTAATGTCA 
Fig. 1 Generation of a Rab39b KO mouse model. a Scheme of the $R a b 39 b$ locus: exons are black boxes spaced from one intron; $5^{\prime}$ - and $3^{\prime}$-UTRs are white boxes. Enlargement of the Rab39b sequence to highlight the target sequence of the MM0000573713 guide (upper), 14 bp deletion (middle; $-14 \mathrm{bp}$ ) and $10 \mathrm{bp}$ insertion (bottom; +10 bp). b Expression profile of Rab39b transcripts in Rab39b WT, KO - 14 bp and $\mathrm{KO}+10$ bp brain lysates $(n=3)$. Data are expressed as $R a b 39 b$ expression normalised to histone $\mathrm{H} 3\left(2^{-\Delta \mathrm{Ct}(\mathrm{Rab} 39 \mathrm{~b}-\mathrm{H} 3)}\right)$. c Representative western blots showing RAB39B protein expression in Rab39b WT, $\mathrm{KO}-14 \mathrm{bp}$ and $\mathrm{KO}+10 \mathrm{bp}$ brain lysates $(n=3)$. Calnexin is the loading reference. d Body weight in grams (g) of Rab39b WT $(n=10)$ and KO $(n=11)$ littermate mice. e Representative images of 20 - and 90-day-old Rab39b WT and KO littermate mice. The scale bar is $2 \mathrm{~cm}$. f Representative MRI scan figures showing abdominal axial "pure fat" obtained by image subtraction (T1 weighted-T1 weighted with fat saturation); after subtraction, the signal of non-fat tissue becomes null. The following main anatomic landmarks were reported: 1-bowel, 2 kidneys, 3-spine and vertebral muscles. The scale bar is $0.5 \mathrm{~cm}$. Graph indicates pure fat in $\mathrm{mm}^{3}$. $\mathbf{g}$ Quantification of the ratio between mature (M) and immature (IM) forms of AMPAR subunits after EndoHf digestion. Lower panels show representative western blots for GluA1 ( $\mathrm{WT}=6, \mathrm{KO}=6, \mathrm{KO}+$ CherryRab39b $=3$ independent lysates), GluA2 (WT $=7, \mathrm{KO}=6, \mathrm{KO}+$ CherryRab39b $=3$ independent lysates) and GluA3 (WT $=6, \mathrm{KO}=6, \mathrm{KO}+$ CherryRab39b $=3$ independent lysates) after PNGasef (P) or EndoHf (E) digestion; ND: nondigested neurons. h Quantification of the neuronal surface expression density of GluA1 (WT $=8, \mathrm{KO}=12$ images), GluA2 (WT $=17, \mathrm{KO}=12$ images) and $\mathrm{GluA} 3$ (WT $=15, \mathrm{KO}=16$ images), expressed in the number of positive puncta/ $\mu \mathrm{m}^{2}$ and representative images. The number of images belongs to a minimum of three experimental replicates. All the data are expressed as the mean \pm SEM. The scale bar is $50 \mu \mathrm{m} .{ }^{*} p<0.05 ; * * p<0.01 ; * * * p<0.001$.

AATAAGAGAAGAC-3') primers [1]. For normalisation, histone $\mathrm{H} 3$ was amplified with mHistone3F (5'-GTGAAG AAACCTCATCGTTACAGGCCTGGTAC-3') and mHistone3R (5'-CTGCAAAGCACCAATAGCTGCACTCTGG AAGC- $3^{\prime}$ ) primers [1].

\section{Metabolism-related parameters}

To measure metabolism-related parameters, P90 Rab39b WT and KO littermate mice were housed individually.

\section{Food and water intake}

Food and water intake evaluation was performed by replacing sawdust with a sheet of $3 \mathrm{~mm}$ paper and $10 \mathrm{~g}$ food pellet in a $\emptyset 6 \mathrm{~cm}$ dish without a lid and giving mice a $15 \mathrm{ml}$ tube full of water. We weighed the $\varnothing 6 \mathrm{~cm}$ dishes and $15 \mathrm{ml}$ tubes with water to calculate the amount of food and water consumed. Food and water intake were measured during light and dark cycles for a total of 2 days. Food intake was measured as the difference in weight between the food put into the cage and that remaining at the end of the dark or light cycle, taking crumbs into account. Water intake was measured as the difference in weight between the $15 \mathrm{ml}$ tube with water and that remaining at the end of the light or dark cycle.

\section{Body temperature}

Body temperature was measured by means of rectal thermometry with the TCAT-2AC Animal Temperature Control (Physitemp, New Jersey, USA) equipped with RET-3 Rectal Probe for Mice.

\section{Glucose evaluation}

The glycaemic index was measured by a Breeze 2 monitoring system (Bayer, Leverkusen, Germany) at the basal level (mice fed ad libitum) and $12 \mathrm{~h}$ after food and water starvation.

$\left[{ }^{18} \mathrm{~F}\right]-\mathrm{FDG}$ was prepared for clinical use following the European Pharmacopeia, VIII edition. Fasting animals were injected with $\left[{ }^{18} \mathrm{~F}\right]-\mathrm{FDG}$ through the tail vein. $1 \mathrm{~h}$ after tracer injection, each animal was anaesthetised by mixing $2 \%$ isoflurane and $1 \%$ medical air; subsequently, animals were sacrificed. Blood samples were collected, and radioactivity was counted in a gamma counter (LKB Compugamma CS1282, Wallac). Radioactivity concentrations in tissue and blood due to radioactive decay were corrected (half-life of $\left[{ }^{18} \mathrm{~F}\right]=108.9 \mathrm{~min}$ ) and expressed as $\%$ of the injected dose per gram of tissue.

\section{Mouse magnetic resonance imaging}

Magnetic resonance imaging was used to measure abdominal adipose tissue and was performed with a dedicated horizontal 7-Tesla scanner (Bruker, BioSpec 70/30 USR, Paravision 5.1, Germany) equipped with a gradient system characterised by an amplitude of 450/675 milliTesla/meter $(\mathrm{mT} / \mathrm{m})$, slew rate of $3400 / 4500 \mathrm{Tesla} / \mathrm{meter} / \mathrm{second}(\mathrm{T} / \mathrm{m} / \mathrm{s})$ and a rise time of 140 milliseconds (ms), coupled with a dedicated volumetric mouse body coil. High-resolution T1weighted sequences with and without fat saturation were acquired covering a body region from the hepatic dome to the inguinal canal, including the scrotum, in male mice. By image subtraction (T1 - T1 fat), a "pure fat" image stack was obtained on which adipose tissue volume was semiautomatically segmented and quantified with region growing function after clustering. Image processing and all the analyses were performed by using open source MIPAV software v.7.0.0 (and later versions) [27].

\section{Hippocampal and cortical cultured neurons, lentiviral transduction, plasmid transfection and NASPM treatment}

Primary neuronal cultures were prepared as in [28] with minor modifications. Hippocampi or cortex were dissected from E18.5 embryos from Rab39b WT and KO females. After 15 min of incubation with $0.25 \%$ trypsin (Euroclone, 
\#ECB3051D) in Hanks' balanced salt solution (HBSS; LifeTech, \#14170-088) at $37^{\circ} \mathrm{C}$, hippocampi or cortex were washed three times with HBSS to remove trypsin and then mechanically dissociated. Neurons were counted, and 150,000 cells were plated on poly-L-lysine (Sigma-Aldrich, \#P2636; $0.2 \mathrm{mg} / \mathrm{ml}$ )-treated glass coverslips (Ø24 mm) for molecular biology experiments or 300,000 cells were plated on poly-L-lysine-treated plastic dishes $(\varnothing 3.5 \mathrm{~cm})$ for biochemical experiments. Cells were plated in NM5 [neurobasal medium (LifeTech, \#21103) supplemented with 5\% foetal bovine serum (FBS; LifeTech, \#16000-034), $2 \mathrm{mM}$ glutamine (Life tech, \#25030-024), $100 \mu \mathrm{g} / \mathrm{ml}$ PenStrep (LifeTech, \#15140) and 2\% B27 (LifeTech, \#17504-044)]. After $2 \mathrm{~h}$ of incubation at $37^{\circ} \mathrm{C}$ and $5 \% \mathrm{CO}_{2}$ to allow adhesion to the substrate, the medium was changed to NM0 (NM5 without FBS).

Transduction was performed at MOI1 with lentiviral particles expressing GFP at 2 days in vitro (DIV) and CherryRab39b at 4 DIV as previously described $[1,13]$.

If transfected, 150,000 neurons/ $/ 24 \mathrm{~mm}$ coverslips were treated at 4 DIV with Lipofectamine 2000 (Invitrogen, \#11668-027) following the manufacturer's instructions with some modifications. In detail, conditioned NM0 medium was collected in a tube to be reused, and $1 \mathrm{ml}$ of prewarmed neurobasal medium was added to the cells and incubated at $37^{\circ} \mathrm{C}$ in a $5 \% \mathrm{CO}_{2}$ humidified atmosphere. In the meantime, solution 1 and 2 were prepared. Solution 1 was prepared by mixing $1 \mu \mathrm{l}$ Lipofectamine 2000 with $100 \mu \mathrm{l}$ Optimem (Life Tech, \#51985) and stored for $5 \mathrm{~min}$ at room temperature. Solution 2 consisted of $0.5 \mu \mathrm{g}$ CherryGluA2 (a gift from Prof. Richard Huganir, Johns Hopkins University School of Medicine, Baltimore, Maryland 21205, USA) mixed in $100 \mu \mathrm{l}$ Optimem. Solutions 1 and 2 were mixed, incubated for $30 \mathrm{~min}$ at $37^{\circ} \mathrm{C}$ and then dispensed on cells. After $1 \mathrm{~h}$ of incubation, the neurobasal medium was replaced with new medium composed of half preconditioned NM0 and half fresh NM0.

If treated, $30 \mu \mathrm{M}$ NASPM was added directly on 150,000 cells/Ø24 mm coverslips without changing the medium, twice a day (at 9 am and $5 \mathrm{pm}$ ) every day from 6 DIV to 13 DIV; then, live imaging was performed at 14 DIV.

\section{De-glycosylation assay}

14 DIV-cultured Rab39b WT, Rab39b KO and Rab39b KO transduced with CherryRab39b [13] hippocampal neurons were lysed with Lysis Buffer [1\% SDS, 2 mM EDTA, $10 \mathrm{mM}$ HEPES $\mathrm{pH} 7,4,10 \mathrm{mM}$ sodium fluoride, $1 \mathrm{mM}$ sodium orthovanadate, $1 \mathrm{mM} \beta$-glicerophosphate, $1 \mathrm{x}$ protease inhibitor cocktail (Sigma-Aldrich, \#P8340)], boiled for $5 \mathrm{~min}$ and syringed eight times. Protein lysates were denatured for $15 \mathrm{~min}$ at $55^{\circ} \mathrm{C}$ and digested with EndoHf (NEB, \#P0703S) or PNGasef (NEB, \#P0704S) for $2 \mathrm{~h}$ at $37^{\circ} \mathrm{C}$. SDS loading buffer $(230 \mathrm{mM}$ Tris- $\mathrm{HCl} \mathrm{pH} 6.8$, $6.85 \%$ SDS, $24 \%$ glycerol, $0.008 \%$ bromophenol blue, $5 \%$ DTT, $5 \% \beta$-mercaptoethanol) was finally added to the samples. Samples were analysed by western blotting.

\section{Western blot}

Mouse primary hippocampal neurons were lysed at 14 DIV in lysis buffer, boiled for $5 \mathrm{~min}$ and syringed eight times. Hippocampi from Rab39b WT and KO mouse brains at 30 days of age $(\mathrm{P})$ were dissected and potterized ten times in homogenisation buffer [ $320 \mathrm{mM}$ saccarose, $5 \mathrm{mM}$ HEPES $\mathrm{pH} 7.4,2 \mathrm{mM}$ EDTA, $10 \mathrm{mM}$ sodium fluoride, $1 \mathrm{mM}$ sodium orthovanadate, $1 \mathrm{mM} \beta$-glycerophosphate, $1 \mathrm{x}$ protease inhibitor cocktail (Sigma-Aldrich, \#P8340)], incubated for $10 \mathrm{~min}$ at $+4{ }^{\circ} \mathrm{C}$ on rotation and then centrifuged at $13,500 \mathrm{~g}$ for $20 \mathrm{~min}$ at $+4^{\circ} \mathrm{C}$.

SDS-PAGE was run with the appropriate percentage of polyacrylamide gel, and then western blots were performed by using appropriate primary and HRP-conjugated secondary antibodies (Supplementary Table 1). Protein bands were detected by a Chemidoc machine (Bio-Rad). ImageJ analysis software (NIH, Bethesda, MD, USA) with the 'Analyse gels' plugin was used to analyse western blots. The pixel size of each protein band was normalised to its relative housekeeping band; for phosphorylated proteins, the ratio between normalised phosphorylated and normalised total forms was also calculated.

\section{Golgi staining}

Golgi staining was performed following the FD Rapid GolgiStain Kit (FD NeuroTechnologies, Columbia, MD, US). Pictures from neuronal apical dendrites in the CA1 region of the hippocampus were captured using an AxioImager: Zeiss AxioImager M2m equipped with a 100x objective [29]. Three mice per genotype and age were analysed, and five dendrites per hemisphere from one slice from each mouse were examined. The number of head spines was manually counted and normalised to a dendritic length of $5 \mu \mathrm{m}$ measured by ImageJ software. The morphology of spines was described as the ratio of spine length and spine width as previously described [30]. A maturity ratio $<2.5$ represented mature mushroom-like spine morphology, a ratio between 2.5 and 6 indicated immature filopodia-like spines, and a ratio $>6$ denoted long spine protrusion.

\section{Immunofluorescence and confocal imaging}

To visualise GFP and Cherry fluorescence, neurons were fixed for $15 \mathrm{~min}$ in fixing solution (4\% paraformaldehyde (Sigma-Aldrich, \#P6148) and 4\% sucrose (Sigma-Aldrich, 
\#S3089) in $120 \mathrm{mM}$ sodium phosphate buffer, $\mathrm{pH}$ 7.4). Coverslips were rinsed three times with PBS and then incubated for $2 \mathrm{~h}$ at RT in a humidified chamber with goat serum dilution buffer (GSDB; $15 \%$ goat serum, $450 \mathrm{mM}$ $\mathrm{NaCl}, 20 \mathrm{mM}$ sodium phosphate buffer, $\mathrm{pH} 7.4)+0.1 \%$ Triton X-100. Coverslips were then mounted with Vectashield (Vectalab, \#H1200) after washing three times within 30 min with high salt buffer (HS: $500 \mathrm{mM} \mathrm{NaCl}, 20 \mathrm{mM}$ sodium phosphate buffer, $\mathrm{pH} 7.4$ ) and one time with $5 \mathrm{mM}$ sodium phosphate buffer, $\mathrm{pH}$ 7.4.

To visualise GluA1, GluA2 and GluA3 exposed on the cell surface [13], cells were incubated for $5 \mathrm{~min}$ with primary antibodies targeting the N-terminal portion of GluA1, GluA2 and GluA3 subunits (Supplementary Table 1) diluted in neurobasal medium at $37{ }^{\circ} \mathrm{C}$ and in $5 \% \mathrm{CO}_{2}$. Then, the cells were fixed in fixing solution and rinsed three times with PBS. Coverslips were incubated for $2 \mathrm{~h}$ at RT in a humidified chamber with the appropriate fluorophoreconjugated secondary antibody (Alexa Fluor, Invitrogen) diluted in GSDB without Triton X-100. Finally, coverslips were mounted with Vectashield (Vectalab, \#H1200) after washing three times within $30 \mathrm{~min}$ with HS and one time with $5 \mathrm{mM}$ sodium phosphate buffer, $\mathrm{pH}$ 7.4. Pictures of hippocampal neurons were captured using a Leica TCS SP8 SMD FLIM laser scanning confocal microscope equipped with an HC PL APO CS 2 63X (NA 1.4) oil-immersion objective. Multiple focal planes with z-spacing of $0.2 \mu \mathrm{m}$ were deconvolved with the Deconvolution wizard of Huygens essential software (SVI, The Netherlands) by providing the lif-file and flattened by ImageJ maximum projection.

AMPAR subunits, relative to the area of GFP and/or Cherry signals, were measured by using the "Gran filter" plugin (circle sizes from 1 to infinity) of ImageJ.

To analyse spines, deconvolved and projected images were Gaussian blurred with ImageJ and imported in Volocity software (Quorum Technologies Inc, Ontario) to manually edit spines, defined as dendritic protuberances from the parent dendrite demarcated by a GFP-soluble signal. Edited spines were analysed by Cell Profiler software (Broad Institute, Cambridge, MA), and eccentricity, compactness, maximum ferret diameter values and skeletonised images were exported for each spine. Spine density was defined as head-spine/ $\mu \mathrm{m}$ dendritic length. Each spine was manually categorised as either having a neck or not a neck. Spine morphology was categorised as follows: spines with necks were separated into thin and mushroom spines based on eccentricity (thin $\geq 0.8$; mushroom $<0.8$ ); spines without necks were divided into filopodia, stubby or blobby spines based on eccentricity, maximum ferret diameter, compactness and the presence of branches defined by the skeletonization process (filopodia: eccentricity $>0.8$, maxferetdiameter $>2.5 \mu \mathrm{m}$; stubby spines were defined as eccentricity $<0.8$ without branches; blobby spines were defined as compactness $>1$ with branches). For presentation of confocal images, contrast was enhanced by linear methods by using ImageJ.

\section{Live imaging}

Live imaging was performed with the samples in KRH

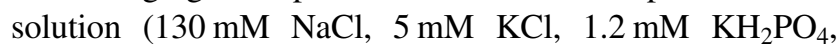
$1.2 \mathrm{mM} \mathrm{MgSO}_{4}, 2 \mathrm{mM} \mathrm{CaCl}_{2}, 25 \mathrm{mM}$ HEPES, $6 \mathrm{mM}$ glucose, pH 7.4, osmolarity 3.08-3.10). 200 frames every $10 \mathrm{~s}$ were recorded $(\sim 33 \mathrm{~min})$ by a Leica SR GSD 3D TIRF equipped with an HC PL APO 160 X (NA 1.4 3) Oil Corr GSD TIRF Pifoc objective and iXon ultra $512 \times 512$ camera. Multiple focal planes (z-spacing $0.21 \mu \mathrm{m}$ ) were deconvolved with the Deconvolution wizard of Huygens essential software. Videos were aligned with the "Registration-Stackreg" plugin of FiJi and imported in Volocity to manually edit spines of $30 \mu \mathrm{m}$ long dendrites and to determine the variation in pixel size of each spine in acquired frames. Values were imported in MATLAB, where spines were categorised as stable spines if present at all times and transient spines if they appeared and disappeared during track recording, and the dynamic rate of each spine was calculated as the pixel size variance of the spine area normalised by the area at time zero [31]. For image presentation, contrast was enhanced by the linear method by ImageJ.

\section{Electrophysiology}

Coronal slices that were $200-250 \mu \mathrm{m}$ thick and that contained the mPFC were prepared from P60 Rab39b WT and $\mathrm{KO}$ mice. Slices were kept for $30 \mathrm{~min}$ in artificial cerebrospinal fluid (aCSF) containing $119 \mathrm{mM} \mathrm{NaCl}, 2.5 \mathrm{mM}$ $\mathrm{KCl}, 1.3 \mathrm{mM} \mathrm{MgCl} 2,2.5 \mathrm{mM} \mathrm{CaCl} 2,1.0 \mathrm{mM} \mathrm{NaH} \mathrm{PO}_{4}$, $26.2 \mathrm{mM} \mathrm{NaHCO}$ and $11 \mathrm{mM}$ glucose that was bubbled with $95 \% \mathrm{O}_{2}$ and $5 \% \mathrm{CO}_{2}$ and then were transferred at room temperature in the same solution. All recordings were made from prelimbic cortex (PrL) layer III. The whole-cell current-clamp recording technique was used to measure spike probability. The pipette internal solution contained (in $\mathrm{mM}$ ) $140 \mathrm{~K}$-gluconate, $2 \mathrm{MgCl}_{2}, 5 \mathrm{KCl}, 0.2$ EGTA, 10 HEPES, 4 $\mathrm{Na}_{2} \mathrm{ATP}, 0.3 \mathrm{Na}_{3} \mathrm{GTP}, 10$ phosphocreatine, $\mathrm{pH} 7.3$ and 300 mOsm. Action potentials (APs) were elicited by injecting small current steps of $50 \mathrm{pA}$ starting at 0 and continuing to 500 pA. APs were counted for each step. To isolate mEPSCs, recordings were made in a voltage-clamp configuration, and neurons were held at $-70 \mathrm{mV}$. $1 \mu \mathrm{M}$ tetrodoxin and $50 \mu \mathrm{M}$ picrotoxin (PTX) were added to the bath solution, and neuronal activity was recorded for $30 \mathrm{~min}$ ( 5 min baseline and 25 min of mEPSCs). mEPSCs were recorded with a Multiclamp 700B amplifier (Axon Instruments, Foster City, CA), filtered at $2.2 \mathrm{kHz}$, digitised at $5 \mathrm{~Hz}$, and analysed using MiniAnalysis 6 (Synaptosoft) software. 
Synaptic plasticity recordings were made in voltageclamp configuration in aCSF $+50 \mu \mathrm{M}$ PTX. Internal pipette solutions contained (in $\mathrm{mM}$ ) $130 \mathrm{CsCl}, 4 \mathrm{NaCl}, 2 \mathrm{MgCl}_{2}$, 1.1 EGTA, 5 HEPES, $2 \mathrm{Na}_{2} \mathrm{ATP}, 5$ sodium creatine phosphate, $0.6 \mathrm{Na}_{3} \mathrm{GTP}, 0.1$ spermine and 5 lidocaine $\mathrm{N}$-ethyl bromide, $\mathrm{pH} 7.3$; osmolarity was adjusted to $289 \mathrm{mOsm}$. EPSCs were elicited by placing a unipolar electrode onto PrL layer 5. Access resistance (10-30 M 2 ) was monitored by a hyperpolarizing step of $-4 \mathrm{mV}$ at each sweep every $10 \mathrm{~s}$. Data were excluded when the resistance changed $>20 \%$. The AMPA/NMDA ratio was calculated by subtracting the mixed AMPA-NMDA EPSC $(+40 \mathrm{mV})$, the AMPA component isolated by D-APV $(50 \mu \mathrm{M}$ at $+40 \mathrm{mV})$ bath application. The rectification index (RI) of AMPARs is the ratio of the chord conductance calculated at a negative potential $(-60 \mathrm{mV})$ divided by the chord conductance calculated at a positive potential $(+40 \mathrm{mV})$. For isolation of AMPA currents sensitive to NASPM, cells were clamped at $-70 \mathrm{mV}$ in aCSF $+50 \mu \mathrm{M}$ PTX, and EPSCs were elicited. After $5 \mathrm{~min}$ (Pre), NASPM $(15 \mu \mathrm{M})$ was added to the recording chamber, and the cells were recorded for $15 \mathrm{~min}$ (Post). The ratio of the Pre-Post response was calculated. The analysis of the decay time of NMDAR-mediated EPSCs was conducted as described previously [32], and ifenprodil sensitivity was calculated as the percentage of NMDAR-EPSC amplitude reduction (at $+40 \mathrm{mV}$ ) after $30-40 \mathrm{~min}$ of continuous ifenprodil $(3 \mu \mathrm{M}$, GluN2Bcontaining NMDAR antagonist) bath application compared to baseline. Synaptic responses were collected with a Multiclamp 700B amplifier (Axon Instruments, Foster City, $\mathrm{CA}$ ), filtered at $2.2 \mathrm{kHz}$, digitised at $5 \mathrm{~Hz}$, and analysed online using Igor Pro software (Wavemetrics, Lake Oswego, OR).

\section{Behavioural tests}

All behavioural tests to compare Rab39b WT and KO littermate male mice were performed on 3- to 4-month-old mice. Animals were video-tracked with the EthoVision 15XT system (http://www.noldus.com) and analysed with Wintrack 2.4 (http://www.dpwolfer.ch/wintrack). For fear conditioning protocols, the ANY-maze system was used (https://www.anymaze.com).

\section{Emergence and novelty test}

Frames of nonreflective aluminium ( $37 \mathrm{~cm}$ high) were used to divide the maze into four arenas $(50 \times 50 \mathrm{~cm})$, allowing concurrent observation of four mice.

For the emergence test, $24 \mathrm{~h}$ before testing, a home box $(12 \times 8 \times 4 \mathrm{~cm})$ with an opened door $(8 \times 4 \mathrm{~cm})$ was inserted into the mouse's home cage. The next day, the home box was placed $5 \mathrm{~cm}$ from the corner of the testing arena with the opened door facing the centre of the arena. Then, the mouse was released in the centre of the arena and tracked for $30 \mathrm{~min}$.

For the novelty test, performed the day after the emergence test, the arena setup was the same as for the emergence test without the home box. The test consisted of two phases of $30 \mathrm{~min}$; in the first phase, the mouse was able to explore the known arena freely, and during the second phase, an object (50 $\mathrm{ml}$ Falcon tube) was vertically glued in the centre of the arena.

Horizontal activity was analysed by segmenting the recorded tracks into three motion states: resting, scanning and progressing. To assess the approach-avoidance conflict towards the arena, three concentric zones were defined: exploration $(55 \%$ of the arena in the emergence tests and $10 \%$ in the novelty tests), transition (29\% of the arena in the emergence tests and 54\% in the novelty tests) and home zones (16\% of the arena in the emergence tests and $36 \%$ in the novelty tests) [33].

\section{Water maze}

Mice were trained in a circular pool of $(\varnothing 150 \mathrm{~cm} ; 50 \mathrm{~cm}$ height) according to a standardised protocol [34]. In the hidden platform $(14 \times 14 \mathrm{~cm})$ version of the water maze, mice had to locate the platform in a fixed position. The test included an acquisition phase (18 trials, 6 per day, intertrial time $30-40 \mathrm{~min}$ ) followed by a reversal phase during which the platform was moved to the opposite position (12 trials, 6 per day). The first $60 \mathrm{~s}$ of trial 19 (the first reversal trial) was considered a probe trial. For the analysis, the trials were averaged in blocks of two trials.

\section{Radial maze}

The apparatus consisted of eight arms with a distance of $47 \mathrm{~cm}$ from a central platform. A cup with a food pellet was placed at the end of each arm. Food-deprived mice (maintained at $85 \%$ of their free-feeding weight) were placed in the centre platform and allowed to collect pellets placed at the end of each arm for $10 \mathrm{~min}$. After 1 day of habituation to the maze, the mice were tested for 10 days. For each trial, the number of correct choices before the first error and the total number of errors were recorded [35].

\section{Spontaneous alternation}

A cross maze was obtained by closing 4 of the 8 arms of the radial maze apparatus. Each mouse was released in the centre platform and allowed to explore the maze for $10 \mathrm{~min}$. The number and sequence of arm entries were recorded throughout the experiment. A correct alternation was considered when no more than one repetition over five entries 
was made. The percentage of correct spontaneous alternations and the total number of visits were calculated [36].

\section{Fear conditioning}

All mice were pre-exposed to the test chamber (Ugo Basile, Italy) for $10 \mathrm{~min}$ on the day preceding the training session (conditioning) to reduce the salience of context cues during conditioning. During the training session of the standard procedure, the trial started with a $1 \mathrm{~min}$ adaptation followed by the presentation of the tone (CS, $15 \mathrm{~s}$ ) superimposed with a foot shock for the last $2 \mathrm{~s}$ (US). In the trace procedure, CS and US phases were separated by a trace $(15 \mathrm{~s})$. For both protocols, CS-US association was repeated five times with $60 \mathrm{~s}$ intertrial intervals (ITIs). The context test and tone test took place $24 \mathrm{~h}$ after conditioning. The context test consisted of 2 min without CS in the same training chamber, and the tone test consisted of $1 \mathrm{~min}$ without CS followed by 1 min with CS in a new environment. During each trial, the frequency of freezing (absence of movements except respiration) was continuously recorded [37].

\section{Statistical analysis}

Graphs and statistical analysis were performed by GraphPad Prism v7 software, except for the analysis of the dynamic rate for which MatLab was used. The Shapiro-Wilk normality test was performed to assess the normal distribution of data. When normally distributed, the data were analysed with independent $t$-tests and repeated measures (RM) ANOVA as appropriate. If the data violated the normality test, the following nonparametric unpaired $t$-tests were performed: Kruskal-Wallis test followed by post hoc Dunn's test or Mann-Whitney U test, as appropriate. Mood's test was used to compare the medians of two samples. For the analysis of variance with two factors (twoway ANOVA, RM two-way ANOVA and RM two-way ANOVA by both factors), normality of sample distribution was assumed, followed by Bonferroni post hoc test.

$P$ values $<0.05$ were considered statistically significant and are detailed in Supplementary Table 2.

\section{Results}

\section{Rab39b KO mice generation and characterisation}

To analyse the pathophysiological contribution of the lack of RAB39B to XLID, Rab39b KO mice were generated by CRISPR/Cas9 technology [24] (Fig. 1a), and Rab39b WT and KO littermate male mice were evaluated. qRT-PCR and western blot analysis of hippocampal Rab39b WT and KO brain lysates showed intact Rab39b mRNA transcripts and loss of RAB39B protein expression in Rab39b KO mice compared to WT mice (Fig. 1b, c).

The evaluation of Rab39b KO mouse body weight, every 7 days from 7 to 91 days (P), showed a $10 \%$ body weight reduction compared to WT, starting in the early stage of development (P7) and that was maintained at each age analysed ( $p=0.0001$; Fig. 1d, e).

To understand whether the decrease in body weight involved the entire organism, all the organs were weighed in young (P20) and adult (P90) mice; no significant differences were observed between genotypes (Supplementary Fig. 1a). Recently, Zhang et al. described macrocephaly in Rab39b KO mice [38] that was not observed in a recent publication about a new mouse model [39]. Because macrocephaly is characterised by extreme overgrowth of cortical brain lengths [26], analysis of cortical measures at P20 and P90 in our Rab39b KO mice did not reveal any differences between genotypes, excluding the macrocephaly phenotype (Supplementary Fig. 1b, c).

To investigate the cause of the loss of body weight in $R a b 39 b$ KO mice, we focused on adult mice (P90). We first monitored food and water intake and body temperature without finding any differences between genotypes (Supplementary Fig. 1d, e). Then, we determined possible variations in circulating blood glucose levels by measuring the glycaemic index (Supplementary Fig. 1g) and glucose consumption in the cortex, striatum, hippocampus, thalamus and cerebellum brain regions by using $\left[{ }^{18} \mathrm{~F}\right]$ Fluoro-2-deoxyd-glucose (FDG) tracer (Supplementary Fig. 1h); no differences were detected between P90 Rab39b WT and KO mice. By magnetic resonance imaging, we revealed that the loss of body weight in Rab39b KO mice could be ascribed to a significant reduction in the volume of abdominal adipose tissue ( $p=0.015$; Fig. 1f).

However, Rab39b KO male mice were viable and fertile, and the mutant allele was transmitted in the expected Mendelian segregation ratio of an X-linked gene.

\section{RAB39B governs GluA2/3 AMPAR trafficking}

Because we previously reported that $80 \%$ RAB39B downregulation by shRNA impairs GluA2/GluA3 AMPAR and ER-to-Golgi trafficking, ultimately affecting the AMPAR composition at the neuronal surface [13], we investigated how the complete deletion of RAB39B impacts GluA1, GluA2, and GluA3 AMPAR subunit secretory pathways and their surface expression.

To analyse the ER-to-Golgi trafficking of AMPAR subunits in Rab39b KO primary hippocampal neurons, $\mathrm{N}$-glycosylation status was analysed. AMPAR subunits are $\mathrm{N}$-glycosylation proteins, and $\mathrm{N}$-glycans from the ER to the Golgi complex are modified to be driven to the plasma membrane. Rab39b WT and KO primary hippocampal 
neurons were digested with Endo-b-N-acetylglucosaminidase $\mathrm{H}$ (EndoH), the enzyme that removes only unmodified $\mathrm{N}$ glycans [40]. By immunoblotting, AMPAR subunits resulted in two isoforms after EndoH digestion, differing in their mobility in gel; these isoforms were an upper band, corresponding to the EndoH-resistant mature isoform, and a lower band, corresponding to the EndoH-sensitive immature isoform. The ratio between the two bands was a measure of subunit maturation (maturity ratio). As a control, neurons were digested with peptide-N-glycosidase F (PNGasef), which removes all N-glycans. We determined that GluA2 and the more striking GluA3 maturity ratios were significantly decreased in Rab39b KO neurons compared to WT neurons (GluA2 $p=0.004$; GluA3 $p<0.0001$; Fig. 1g), suggesting ER protein retention. In contrast, the GluA1 maturity ratio showed an increase ( $p=0.02$; Fig. $1 \mathrm{~g}$ ). Transducing Rab39b $\mathrm{KO}$ neurons with lentiviral particles expressing CherryRab39b did not affect the total amount of AMPAR subunits (Supplementary Fig. 1i), whereas GluA1, GluA2 and GluA3 maturity ratios were completely restored to those of WT conditions ( $R a b 39 b \mathrm{KO}$ vs Rab39b $\mathrm{KO}+$ CherryRab39b; GluA1 $p=0.05$, GluA2 $p=0.004$, GluA3 $p<0.0001$; Fig. 1g). Because alteration of the AMPAR subunit secretory pathway alters receptor surface expression [13], we next examined AMPAR surface density by immunolabelling $R a b 39 b$ WT and KO hippocampal neurons lacking permeabilization. We showed decreased GluA2 and GluA3 surface density and increased GluA1 surface density on Rab39b KO neurons compared to the surface density observed on WT neurons (GluA1, GluA2 and GluA3 $p<0.0001$; Fig. 1h).

Together, these data indicated that the complete lack of RAB39B specifically impairs GluA2/GluA3 heterotetrameric trafficking, regulating cell surface expression. Moreover, the increase in GluA1 maturation, not observed under downregulation conditions [13], with a concomitant increase in surface expression, suggests increased trafficking of homomeric GluA1 AMPAR.

\section{RAB39B is critical in spine development}

The coordination of GluA2-AMPAR subunit expression and targeting during neuronal development participates in synaptic network establishment through precise dendritic spine morphological reorganisation of excitatory neurons [41, 42], and alterations in AMPAR composition are generally observed in young animals; these alterations are associated with immature synapses and cognitive impairment $[16,43]$. IDs together with a broad spectrum of neurodevelopmental disorders are characterised by defects in spine morphogenesis and/or refinement, often resulting in abnormal dendritic spine number and morphology [44]. We also determined whether the lack of RAB39B might influence dendritic spine development by analysing the spine density (Fig. 2a, b) and morphology (Fig. 2a, c) of apical dendrites of glutamatergic neurons in the CA1 area of the hippocampus of Rab39b WT and KO brains by Golgi staining. Analysis was performed at salient time points of hippocampal neuronal development at P20, P30 and P90, corresponding to spinogenesis [19], synaptic pruning [45] and spine maintenance [21], respectively (Fig. 2a).

In agreement with what was previously described [46], during development, Rab39b WT spines underwent a significant reorganisation of their density (P20 vs P30 vs P90: $p<0.0001$; Fig. 2b) and their morphology (mature, immature, long protrusions $p<0.0001$; Fig. 2c). The evaluation of $R a b 39 b \mathrm{KO}$ hippocampi revealed that no reorganisation in spine density and morphology occurred and that spines remained in a P20-like scenario (Fig. 2a-c).

Moreover, investigation of the genotype effect on the developmental time points did not show differences in $R a b 39 b$ WT and KO neurons in spine density and morphology at P20 but differences were observed at P30 and P90 (density: P30 $p<0.0001$, P90 $p=0.0003$; morphology: mature, immature, long protrusions P30 $p<0.0001$, mature, immature, long protrusions P90 $p<0.0001$; Fig. 2b, c). These data suggest that RAB39B is not essential for the early stages of spine development, such as spinogenesis, but it is crucial during spine pruning and refinement periods.

To address the hypothesis that GluA2-AMPAR subunit RAB39B-mediated trafficking is required to guarantee spine reorganisation, we investigated the impact of RAB39B on spine pruning and remodelling. In primary hippocampal neurons, spine pruning and remodelling occur between 7 and 9 DIV, eliciting final neuronal maturation at 14 DIV [47], according to in vivo brain development (Fig. 2a) $[21,45]$. We performed in vitro rescue experiments by reexpressing RAB39B or GluA2 at 4 DIV-before the gross morphological changes occurred between 7 and 9 DIV-to evaluate the in vitro long-term fate of Rab39b KO hippocampal spines at 14 DIV.

After validation of proper in vitro spine pruning and remodelling of WT spines comparing Rab39b WT spines between 7 and 14 DIV (density $p=0.0021$; morphology: mushroom $p=0.0001$, thin, filopodia, blobby $p<0.0001$; Fig. 2d-f), we also confirmed an immature spine arrangement of Rab39b KO spines at 14 DIV that were comparable to WT at 7 DIV but were significantly different from WT at 14 DIV (density $p<0.0001$; morphology: mushroom, thin, blobby $p<0.0001$, filopodia $p=0.0003$; Fig. 2d-f).

The expression of CherryRab39b or CherryGluA2 in Rab39b KO hippocampal neurons revealed that spine reorganisation was fully recovered by reintroducing RAB39B (density $p=0.008$; morphology: mushroom $p<$ 0.0001 , thin $p=0.03$, filopodia $p=0.02$, blobby $p=0.002$; Fig. 2d-f) but not GluA2 (Fig. 2d-f). In line with the predicted model, GluA2-AMPAR surface expression was 

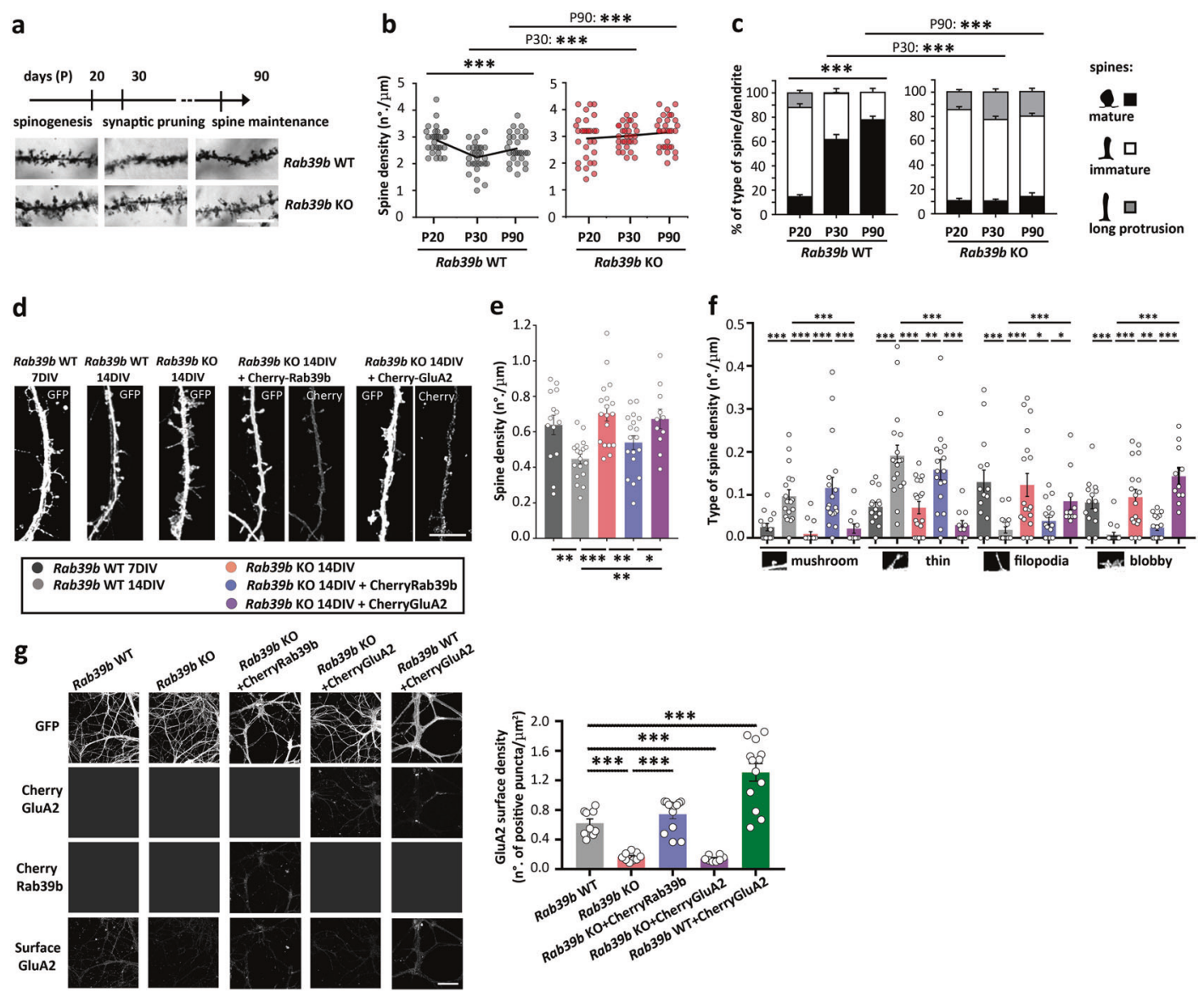

h
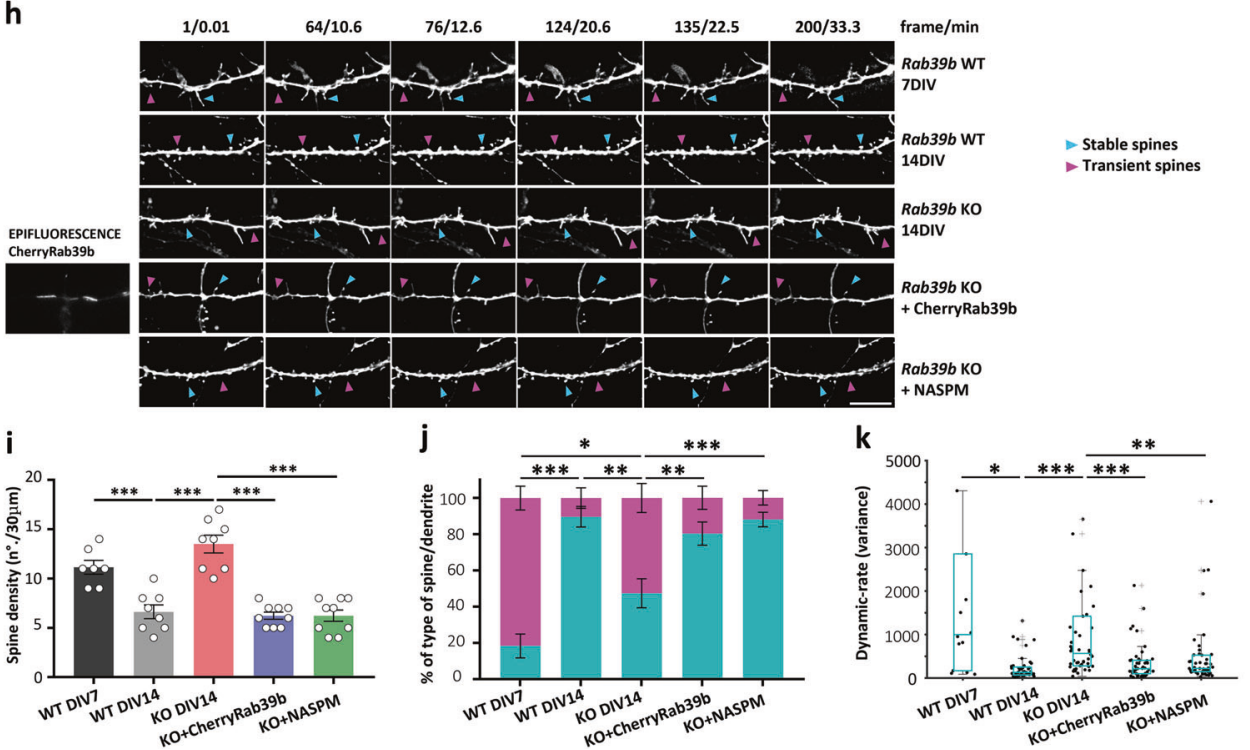

restored only by reintroducing RAB39B in $R a b 39 b \mathrm{KO}$ hippocampal neurons but not GluA2 (Rab39b WT vs KO $p<0.0001 ;$ Rab39b KO vs KO + CherryRab39b $p<$ $0.0001 ; R a b 39 b$ WT vs KO + CherryGluA2 $p<0.0001$; Fig. 2g). As a control to exclude possible defects in CherryGluA2 plasmid expression, CherryGluA2 was expressed in WT neurons, showing an increased GluA2 surface density compared to WT neurons (Rab39b WT vs WT + CherryGluA2 $p=0.0002$; Fig. $2 \mathrm{~g}$ ).

In conclusion, RAB39B-mediated vesicle trafficking is necessary and sufficient for spine elimination and remodelling towards a mature spine state. 
Fig. 2 RAB39B-driven trafficking controls spine refinement. a Timeline of hippocampal dendritic spine development: spinogenesis at 20 days $(\mathrm{P})$, synaptic pruning at $\mathrm{P} 30$ and spine maintenance at $\mathrm{P} 90$. Representative images of Golgi-impregnated CA1 hippocampal apical dendrites of Rab39b WT and KO mice $(n=3)$. The scale bar is $10 \mu \mathrm{m}$. b Spine density in number of spine heads/ $\mu \mathrm{m}$. c Partitioning of spine morphology along a dendrite in \% of type of spine/dendrite. d Representative fluorescence images of $R a b 39 b$ WT and KO primary hippocampal neurons fixed at $14 \mathrm{DIV}$ and/or 7 DIV and Rab39b KO neurons transduced with CherryRab39b or transfected with CherryGluA2 (WT 7 DIV = 14; WT 14 DIV = 18; KO 14 DIV = 18; KO 14 DIV + CherryRab39b $=18 ;$ KO 14 DIV + CherryGluA2 $=11$ dendrites from three independent preparations). The scale bar is $5 \mu \mathrm{m}$. e Spine density as the number of spine heads $/ \mu \mathrm{m}$. f Spine morphology as the type of spine/ $\mu \mathrm{m}$. $\mathrm{g}$ Representative images of primary hippocampal neuronal dendrites and quantification of the neuronal surface expression density of GluA2 as the number of positive puncta/ $\mu \mathrm{m}^{2}$ of cultured GFP-transduced Rab39b WT ( $n=9$ images), KO ( $n=9$ images), KO transduced with CherryRab39b ( $n=13$ images) or transfected with CherryGluA2 ( $n=8$ images) and WT transfected with CherryGluA2 ( $n=13$ images). Images are from at least three independent experiments. The scale bar is $50 \mu \mathrm{m}$. h Representative live imaging frames of primary hippocampal neuronal dendrites of GFPtransduced Rab39b WT 7 DIV $(n=7)$ and 14 DIV $(n=8)$, KO 14 DIV $(n=7), \mathrm{KO}$ transduced with CherryRab39b $(n=9)$ and KO after NASPM treatment $(n=9) . n$ dendrites are from at least three independent experiments. Examples of spine behaviour: stable spines in cyan and transient spines in magenta arrows. The scale bar is $10 \mu \mathrm{m}$. i Spine density expressed as spine-head $/ 30 \mu \mathrm{m}$ dendritic length. $\mathbf{j} \%$ of stable or transient spines/dendrite. $\mathbf{k}$ Dynamic rate as the pixel size variance within the stable spines. Data are the mean \pm SEM except for (k), where the box-central mark is the median, the 25th and 75th percentiles are bottom and top edges, and ' + ' are outliers. $* p<0.05$, $* * p<0.01, * * * p<0.001$

\section{RAB39B deficiency leads to increased spine dynamics, rescued by acting on GluA2-lacking AMPARs}

Having assessed the essential role of RAB39B-mediated trafficking in the orchestration of dendritic spine maturation, it was still not clear how RAB39B influences correct spine remodelling; does the absence of $\mathrm{RAB} 39 \mathrm{~B}$ freeze the spine in an immature state or does it increase the spine dynamic by not allowing it to stabilise itself in a mature state?

A live imaging technique was used to evaluate the spine dynamics of in vitro hippocampal neurons over time (Fig. 2h-k).

First, we determined how WT spines typically behave in vitro during development (at 7 and 14 DIV). A decrease in spine density ( $p=0.0006$; Fig. $2 \mathrm{i}$ ) validated the previous results (Fig. 2e) other than an increase of $70 \%$ stable spines, and a concomitant decrease in transient spines from 7 DIV to 14 DIV was observed (stable, transient $p=0.0003$; Fig. 2j). In addition, the dynamic rate of stable spines significantly decreased from 7 to 14 DIV ( $p=0.014$; Fig. $2 \mathrm{k}$ ), indicating that WT spines moved from an immature hyperdynamic state at 7 DIV to a mature and more stable arrangement at 14 DIV.
Following the Rab39b KO spine dynamic, spine density significantly increased compared to that of WT at 14 DIV $(p<0.0001$; Fig. 2i), as also shown in Fig. 2e; moreover, regarding WT spines, 50\% were transient spines and 50\% stable were spines corresponding to an intermediate state between 7 DIV and 14 DIV (KO 14 DIV vs WT 7 DIV: stable, transient $p=0.017$; KO 14 DIV vs WT 14 DIV: stable, transient $p=0.0017$; Fig. $2 \mathrm{j}$ ). Given the above results that defined Rab39b KO 14 DIV spines as immature and comparable to WT spines at 7 DIV (Fig. 2d-f), we expected to find more correspondence of spine behaviour between Rab39b KO at 14 DIV and WT at 7 DIV. Detailing the Rab39b KO-stable spine dynamic behaviour, these spines exhibited a dynamic rate comparable to WT spines at 7 DIV, but not to WT spines at 14 DIV ( $p=4.17 \mathrm{E}-07$; Fig. 2k), endorsing the immature behaviour of Rab39b KO dendritic spines.

To assess the direct correlation between the role of RAB39B and spine stabilisation, we reintroduced RAB39B by transducing Rab39b KO hippocampal neurons at 4 DIV with CherryRab39b and then analysed them at 14 DIV. Spine density (KO 14 DIV vs KO + CherryRab39b $p<0.0001$; Fig. 2i) and spine dynamics (KO 14 DIV vs KO + CherryRab39b, stable, transient $p=0.009$; dynamic rate $p=8.49 \mathrm{E}-04$; Fig. $2 \mathrm{j}, \mathrm{k}$ ) were rescued so that they were comparable to WT 14 DIV conditions.

The nature of $R a b 39 b$ KO dendritic spines may be ascribed to robust $\mathrm{Ca}^{2+}$ entry caused by increased $\mathrm{Ca}^{2+}$ permeable AMPAR surface expression (Figs. $1 \mathrm{~h}$ and 31 ) and by the absence of GluA2-RAB39B-mediated trafficking (Figs. $1 \mathrm{~g}, \mathrm{~h}$ and $2 \mathrm{~g}$ ). Rab39b KO primary hippocampal neurons were treated with NASPM, a validated $\mathrm{Ca}^{2+}$. permeable AMPAR antagonist [48], between 6 DIV and 13 DIV, before spine stabilisation occurred. Live imaging at 14 DIV showed that NASPM treatment completely restored the dynamics of Rab39b KO spines so that they were comparable to the WT condition (KO 14 DIV vs KO+ NASPM, stable, transient $p<0.0001$; dynamic rate $p=$ 0.001; Fig. $2 \mathrm{~h}-\mathrm{j}$ ).

Our previous and present data thus far has demonstrated that the complete lack of RAB39B impairs GluA2/GluA3 trafficking, altering the availability of a correct AMPAR composition at the neuronal surface and determining neuronal $\mathrm{Ca}^{2+}$ permeability, which is directly responsible for dendritic spine turnover towards a mature synaptic state, as shown by RAB39B reintroduction in a KO environment or by NASPM treatment.

We also evaluated possible variations in the total amount of proteins involved in the direct modulation of spine formation, maturation and stabilisation [49-53], and no differences were detected between genotypes (Supplementary Fig. 2). 
a

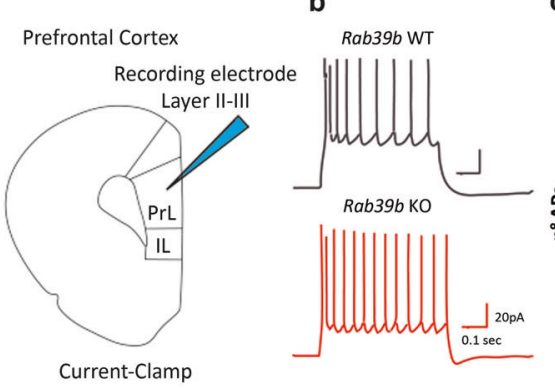

c

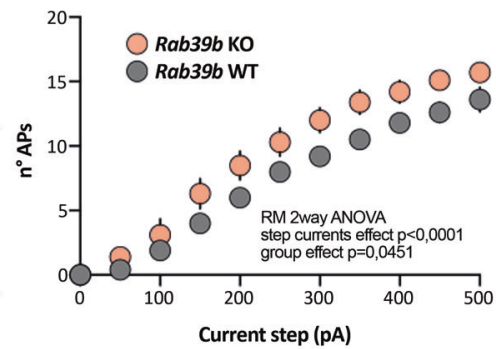

d

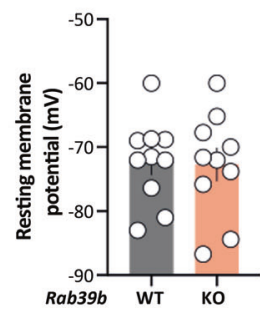

e

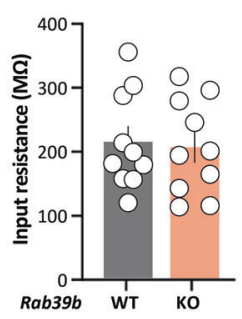

f
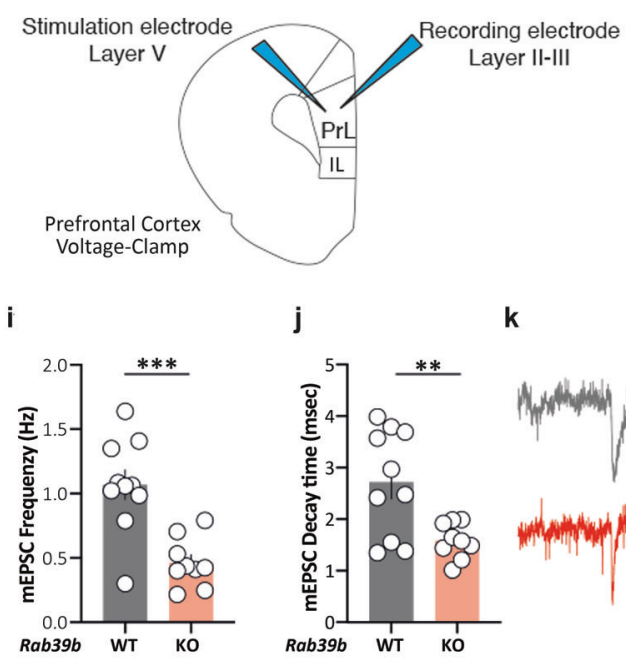

j

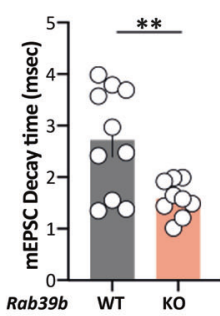

g

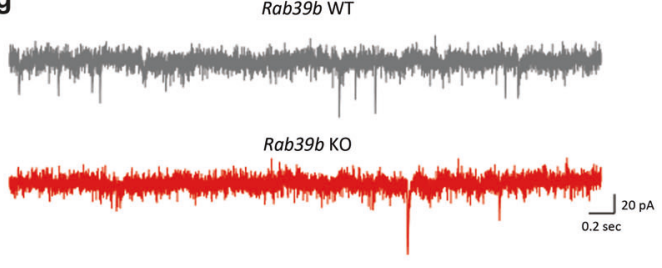

h

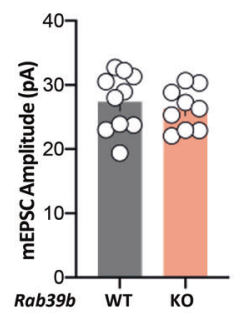

k

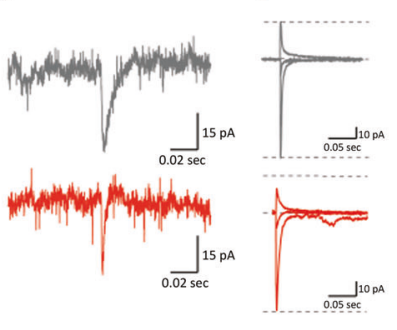

m

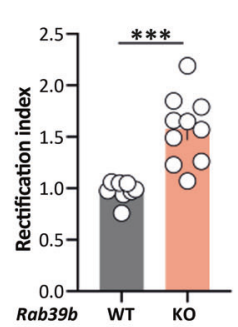

n

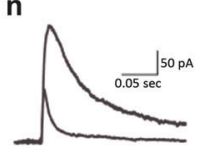

o
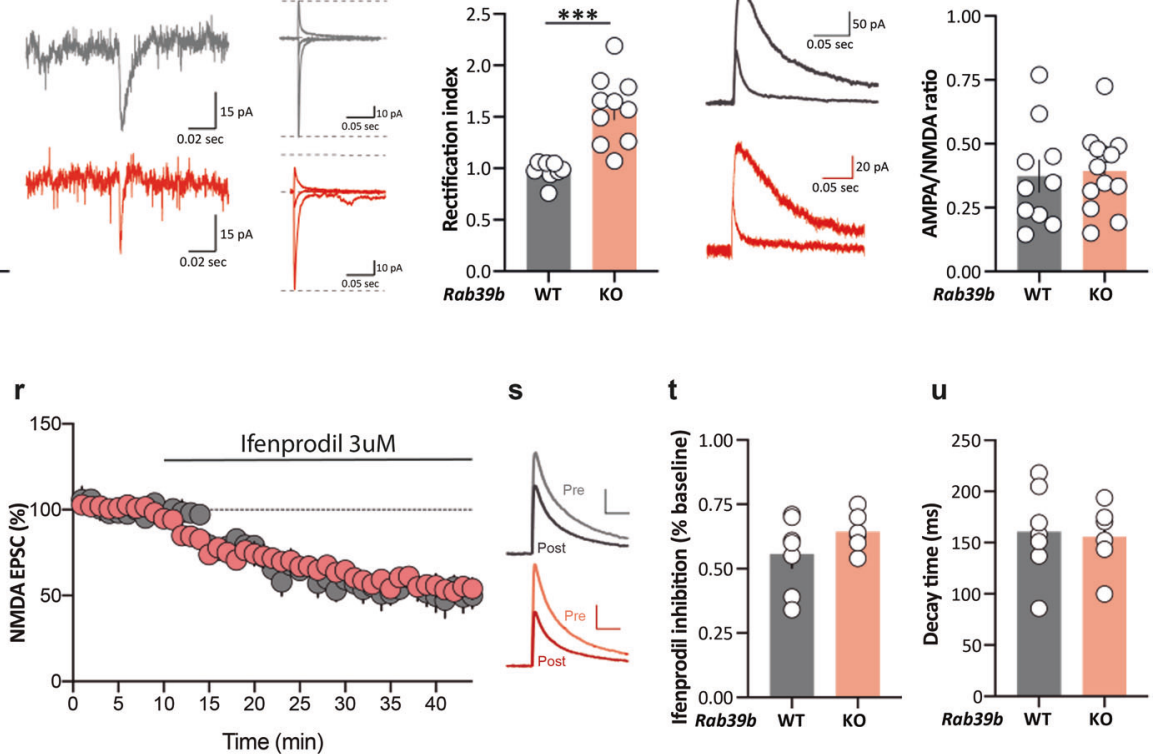

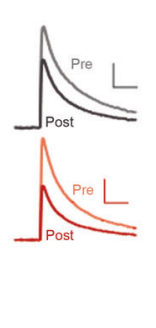

t

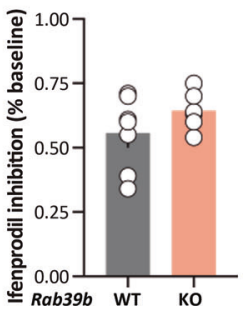

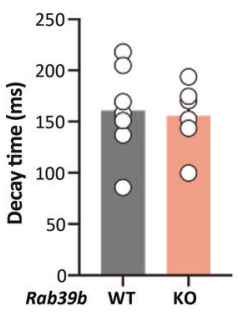

Fig. 3 The lack of RAB39B impairs neuronal function. a Diagram of a patched region. $\mathbf{b}$ Example traces of pyramidal neuron excitability. c Summary data of the number of APs in response to $50 \mathrm{pA}$ current steps. d Scatter plot of the resting membrane potential. e Scatter plot of the input resistance. f Diagram of a patched and stimulation region. g Example traces of mEPSCs. h Scatter plot of mEPSC amplitude. i Scatter plot of mEPSC frequency. $\mathbf{j}$ Scatter plot of mEPSC decay time. $\mathbf{k}$ Example traces of AMPA current decay time. 1 AMPAR-EPSCs example traces $(-60,0$, and $40 \mathrm{mV})$. $\mathbf{m}$ Scatter plot of the rectification index recorded from cortical pyramidal neurons. $\mathbf{n}$ AMPAR/NMDA example traces at $+40 \mathrm{mV}$. o Scatter plot of the AMPA/NMDA ratio

\section{The lack of RAB39B impairs neuronal function}

It has been shown that during development, there is a switch in AMPA subunit composition. Indeed, cortical pyramidal recorded from cortical pyramidal neurons. p Scatter plot of AMPA currents sensitive to NASPM. q AMPA EPSC example traces before and after NASPM bath application. $\mathbf{r}$ Time course of NMDA EPSCs in the presence of ifenprodil (WT mice in grey, Rab39b KO mice in red). s NMDA EPSC example trace before and after ifenprodil bath application. $\mathbf{t}$ Scatter plot of ifenprodil inhibition calculated as $\%$ of baseline between 30 and $40 \mathrm{~min}$ after ifenprodil bath application. u Scatter plot of NMDA EPSC decay time before ifenprodil bath application. All the data are expressed as the mean \pm SEM. $* p<0.05 ; * * p<0.01$; ***p $p<$ 0.001 . For each experiment $3 \mathrm{KO}$ and $3 \mathrm{WT}$ mice were used.

neurons express GluA2-lacking AMPARs during early postnatal development when spinogenesis is at its maximum [54, 55]. We examined whether the absence of $\mathrm{RAB} 39 \mathrm{~B}$ could give rise to alterations in intrinsic electrical 
properties and excitatory synaptic transmission to cortical pyramidal neurons, that showed similar dendritic spine morphological alterations compared to WT (density $p=$ 0.014; morphology: mushroom $p=0.0001$, thin $p=$ 0.0004 , filopodia $p=0.034$, blobby $p<0.0001$; Supplementary Fig. 3) as Rab39b KO hippocampal neurons (Fig. 2d-f). Using a whole-cell patch clamp configuration, we first assessed the spike frequency of cortical pyramidal neurons in response to steps of current injections. As shown in Fig. $3 \mathrm{~b}$ and c, Rab39b KO neurons had increased excitability compared to WT Rab39b neurons $(p=0.045)$ without a change in the resting membrane potential (Fig. 3d), or in the input resistance (Fig. 3e). We next performed recordings of AMPA miniature excitatory postsynaptic currents (AMPA-mEPSCs) in Rab39b WT and KO neurons. Under these conditions, we detected no differences in AMPA-mEPSC amplitude (Fig. 3h) but noted a decrease in the frequency ( $p=0.0003$; Fig. 3i) and decay time $(p=$ 0.0062 ; Fig. 3j, k) in Rab39b KO neurons compared to WT neurons. We then evaluated whether the absence of RAB39B could alter synaptic strength in Rab39b KO neurons synapsing onto pyramidal cortical neurons by recording excitatory postsynaptic currents (EPSCs). We found an increase in the RI $(p<0.0001$; Fig. 31, m) and no changes in the AMPA/NMDA ratio (Fig. 3n, o). When we bath applied NASPM, a selective antagonist of $\mathrm{Ca}^{2+}$ permeable AMPA receptors, we found that pyramidal neurons recorded from KO mice showed a greater decrease in the AMPA amplitude than neurons recorded from WT mice, thus confirming the insertion of GluA2-lacking AMPA receptors $(p=0.0049$, Fig. 3p, q). Subsequently, we tested ifenprodil sensitivity in pharmacologically isolated NMDA currents. We did not detect differences in ifenprodil inhibition (Fig. 3r-t) or in basal decay time kinetics (Fig. 3u), suggesting no alterations in NMDA receptor subunit composition. Together, these results suggest that the absence of RAB39B does not alter synaptic strength but increases the proportion of GluA2-lacking AMPARs in synapses, suggesting a lack of physiological switching of the AMPA receptor.

\section{Rab39b KO mice showed increased activity and impaired associative memory}

To evaluate possible behavioural alterations comparable to those observed in ID features [1, 4], 3- to 4-month-old $R a b 39 b$ WT mice and KO littermate adult male mice were subjected to a battery of behavioural tests to evaluate emotional and explorative behaviour and cognitive impairment.

We examined explorative and emotional behaviour using emergence (Fig. 4a, c, e, g, i) and novelty (Fig. 4b, d, f, h, j) tests [33].
In the emergence test, locomotor activity, scored by total path and velocity, was significantly increased in $R a b 39 b$ KO mice, as shown by a significant effect of path while progressing $(p<0.0001)$, for velocity during scanning $(p=0.013)$ and during progression $(p<0.0001)$ (Fig. $4 \mathrm{a}, \mathrm{c})$. These results were mirrored by a significant decrease at rest compared to the increase observed during progression (rest: $p=0.0006$; progression: $p<0.0001$; Fig. 4e).

In the novelty test, scanning activity was significantly increased in Rab39b KO mice, whereas progression was decreased (scan: $p<0.0001$; progression: $p<0.0001$; Fig. 4b) with no changes in velocity (Fig. 4d). Here, the absence of increased activity in Rab39b KO mice relied on the presence of an object in a yet-known arena, as shown by the significant difference observed in the percentage of time in rest compared to the increase observed during scanning and progression caused by the presence of an object (rest, scan, progression: $p<0.0001$; Fig. $4 \mathrm{f}$ ).

Mice approached and explored novel stimuli and the environment, but they avoided unfamiliar environments or objects by exhibiting fear-related responses. In the emergence test, the home box was a small familiar zone within a large unfamiliar arena, whereas in the novelty test, the novel stimulus was an object placed in the centre of an already familiar arena. Moreover, approach or avoidance in mice reflected the arena zone preferences of the mice (pictograms in Fig. 4g, h).

In the emergence test, Rab39b KO mice significantly preferred to stay in the less aversive home zone $(p=0.004$; Fig. 4g) without hiding themselves in the home box compared to WT mice (Fig. 4i).

Contrastingly, in the novelty test, a significant difference between genotypes was observed in lack of avoidance of exploration $(p=0.0005)$ and transition $(p=0.0006)$ zones concomitant with a lower preference for the home zone $(p<$ 0.0001 ; Fig. 4h). This phenotype of Rab39b KO mice was justified by an excess of object approaches without losing interest during the $30 \mathrm{~min}$ observation period $(p=0.010$; Fig. $4 \mathrm{j})$.

Rab39b KO mice had increased activity in the emergence test compared to control animals and curiosity towards novelty, as shown by increased object investigation.

We then examined learning and memory. In the water maze task [34], Rab39b KO mice were indistinguishable from WT mice, suggesting no deficit in spatial memory (Supplementary Fig. 4).

In the eight-arm radial maze in which mice were to perform a spatial short-term working memory task (Fig. 5a-c), both Rab39b WT and KO mice showed a significant decline in the number of total errors over the 10 days of training, reflecting their ability to patrol the maze (WT: $p<0.0001$; KO: $p<0.0001$; Fig. 5 b), and no 
Fig. 4 Increase activity and curiosity towards novelty in Rab39b KO. Comparison of $(\mathbf{a}, \mathbf{c}, \mathbf{e}, \mathbf{g}$, and i) emergence (WT $=20, \mathrm{KO}=21$ mice) and $(\mathbf{b}, \mathbf{d}, \mathbf{f}, \mathbf{h}$, and $\mathbf{j})$ novelty (WT $=$ $12, \mathrm{KO}=14$ mice) tests for several variables. a, b Distance travelled in metres. $\mathbf{c}, \mathbf{d}$ Velocity in metres/second. e, $\mathbf{f} \%$ of time spent in the three motion states: resting, scanning and progressing. $\mathbf{g}, \mathbf{h} \%$ of time in exploration (E), transition (T) and home $(\mathrm{H})$ zones, highlighted in black in the pictograms below the graphs. i \% of time spent in the home box ( $\mathrm{WT}=8, \mathrm{KO}=$

7). $\mathbf{j}$ Distance to the object while in the exploration zone in centimetres. Data are presented as the mean \pm SEM. $* p<0.05$; $* * p<0.01 ; * * * p<0.001$.
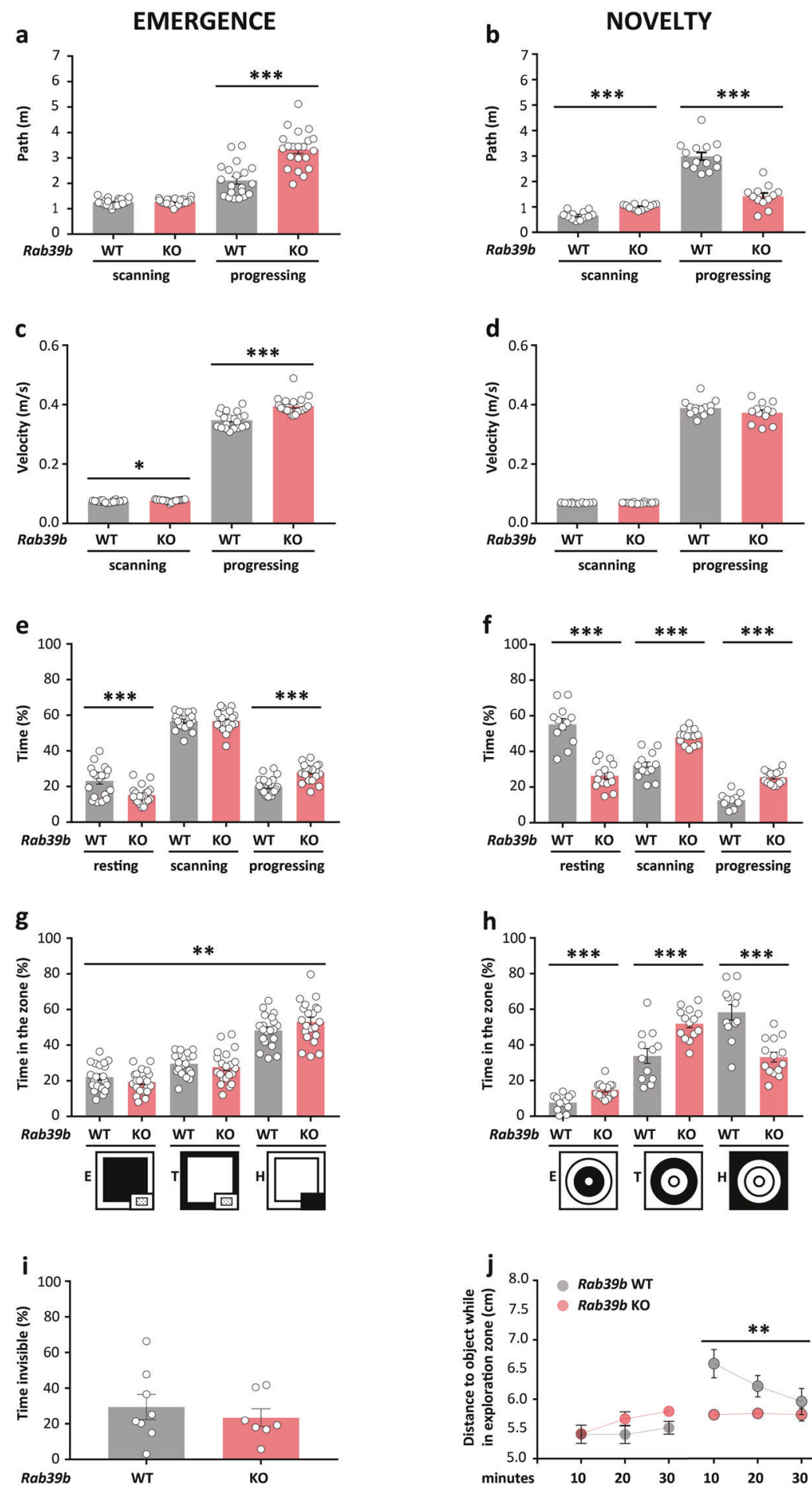

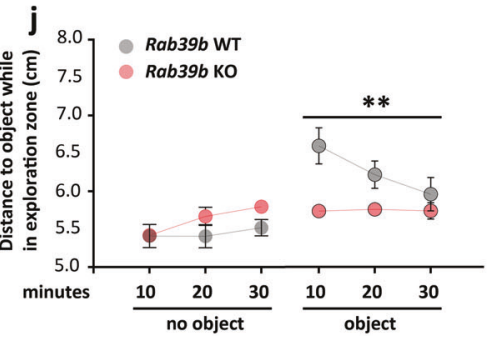

difference was observed in the number of errors (Fig. 5b). Rab39b KO mice significantly differed from WT mice in correct responses ( $p=0.05$; Fig. $5 c$ ). Though WT mice had good performance $(7 \pm 0.18$ out of 8 correct responses, Fig. 5c), KO mice did not exceed the chance level of 5.5 correct responses after 10 days of training $(5 \pm 0.32$ out of 8 correct responses, $p<0.0001$; Fig. 5 c).
A similar deficit was observed in the spontaneous alternation task, a second working memory test (Fig. 5d-f), in that no differences were found in the number of visits (Fig. 5e) but the percentage of correct alternations was significantly different (Rab39b WT $54 \pm 3 \%$ and KO $44 \pm$ $3 \% ; p=0.04$; Fig. $5 \mathrm{f}$ ); this finding suggests that a lack of RAB39B impairs short-term working memory. 
a

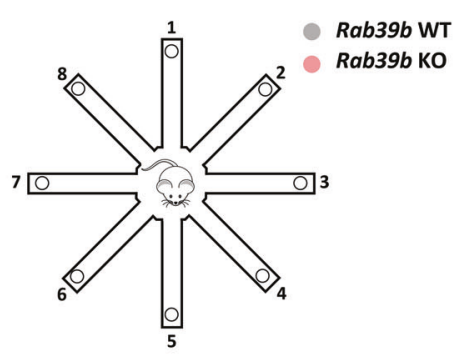

d

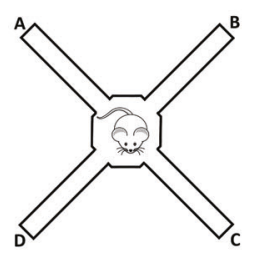

g
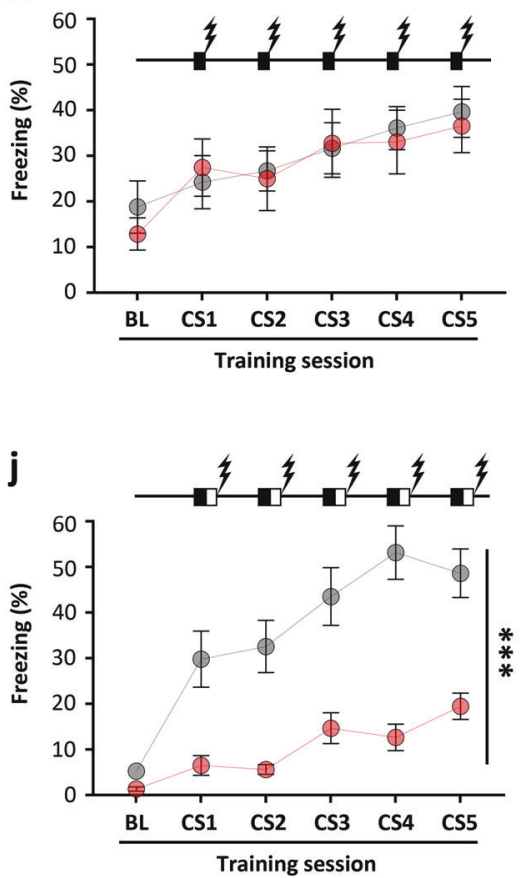

b
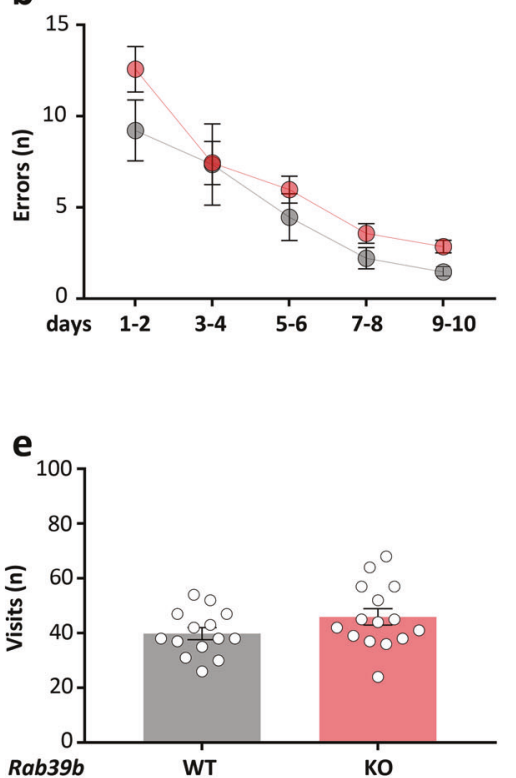

h

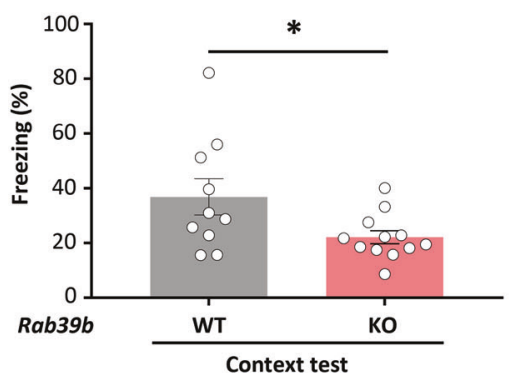

k

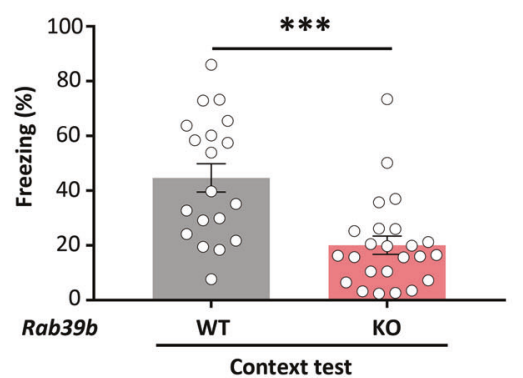

c

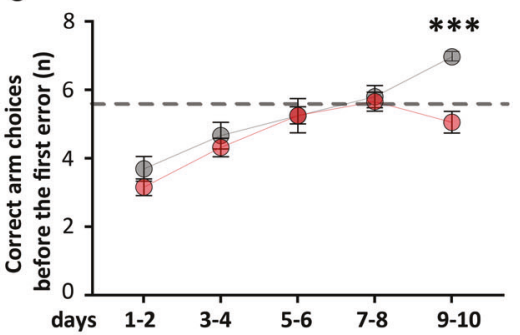

f

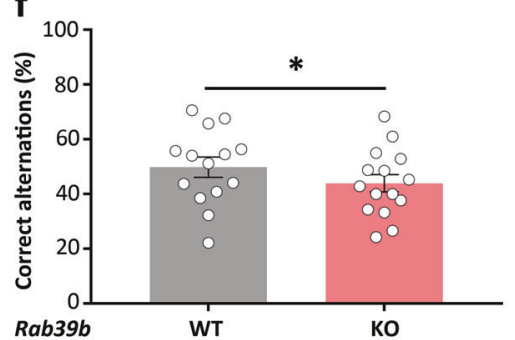

i

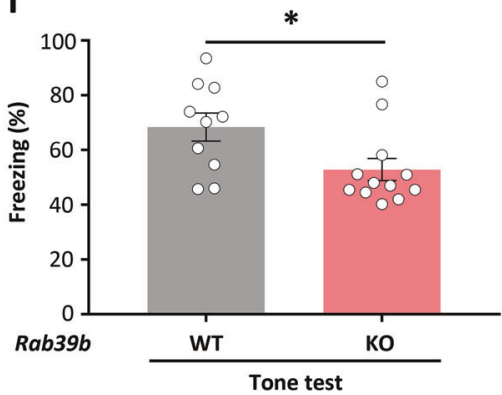

I

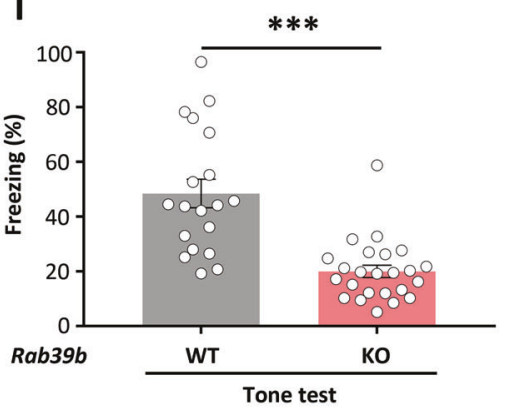

Fig. 5 Rab39b KO mice are impaired in working and associative memory. a Pictogram of the 8-arm radial maze apparatus. Graphs indicate (b) the number or errors and (c) the number of correct arm choices before the first error during the 8 -arm radial maze test (WT $=$ $18, \mathrm{KO}=25$ mice). The dashed line is the chance level performance of 5.5 correct successive arm visits. d Pictogram of the spontaneous alternation apparatus. Graphs show (e) the number of visits and (f) the $\%$ of correct alternations in the spontaneous alternation test $(\mathrm{WT}=14$, $\mathrm{KO}=15$ mice). $\mathbf{g}-\mathbf{i}$ Standard and $(\mathbf{j}-\mathbf{l})$ trace fear conditioning protocols (standard: $\mathrm{WT}=10, \mathrm{KO}=12$; trace: $\mathrm{WT}=19, \mathrm{KO}=24$ mice). $\mathbf{g}, \mathbf{j} \%$ of freezing elicited by repeated exposure to the conditioned stimulus (CS) during the training session. BL indicates the baseline. Pictograms on the graphs represent (g) the standard fear conditioning protocol where the $15 \mathrm{~s}$ tone (CS, black box) is superimposed with a foot shock (lightning) for the last $2 \mathrm{~s}$ and (j) the trace fear conditioning protocol where the CS and the foot shock are separated by a $15 \mathrm{~s}$ trace (white box). (h, i, k, l) \% of freezing during the memory test, $24 \mathrm{~h}$ after training, $(\mathbf{h}, \mathbf{k})$ for context, and (i, l) for tone. Data are presented as the mean \pm SEM. $* p<0.05, * * * p<0.001$. 
We finally assessed associative learning by auditory fear conditioning. In the training session of the standard procedure, no difference between genotypes in the percentage of freezing was detected (Fig. 5h), but during context and tone memory, Rab39b KO mice significantly differed from WT mice (context: $p=0.036$; tone: $p=0.026$; Fig. $5 \mathrm{~h}, \mathrm{i}$ ). In trace fear conditioning, Rab39b KO mice froze significantly less than WT mice during conditioning ( $p<0.0001$; Fig. 5j) as well as in the subsequent two test sessions (context: $p<$ 0.0001 ; tone: $p<0.0001$; Fig. $5 \mathrm{k}, 1)$. Both fear conditioning protocols revealed that the loss of RAB39B impairs associative memory.

\section{Discussion}

As a follow-up to our previous work [13] and taking advantage of the Rab39b KO mouse model that we generated, we determined that the complete absence of RAB39B leads to a more excitable synaptic network and retained $\mathrm{Ca}^{2}$ ${ }^{+}$-permeable AMPARs at synapses. This condition is directly associated with immature spines, which remain hyperdynamic and fail to prune at the postnatal stage. These altered neuronal circuits are mirrored in several behavioural traits, such as hyperactivity and memory deficits reminiscent of the human ID condition.

The remarkable inference from this study is that the aberrant expression of GluA2-lacking AMPARs in adult $R a b 39 b$ KO synapses defined by the AMPAR maturation index, by AMPAR surface expression, and by the increase in the RI in Rab39b KO pyramidal cortical neurons as indicated by electrophysiological data at the functional level, is an important mechanism that regulates dendritic spine development. Indeed, this suggests that the canonical switch from GluA2-lacking to GluA2-containing AMPARs, characterising neuronal development [55], does not occur in Rab39b KO mice. GluA2-lacking receptors are typically $\mathrm{Ca}^{2+}$ permeable; thus, their activation induces an influx of calcium, which in turn could affect neuronal excitability. When directly injected with current, we found an increase in neuronal excitability in cortical pyramidal neurons of Rab39b KO mice compared to WT mice. This suggests that the absence of RAB39B could have a role in the regulation of neuronal intrinsic properties, and whether this is a direct or indirect effect needs to be further investigated. Moreover, though the absence of RAB39B does not alter either the synaptic strength or the NMDA subunit composition, we found that it induces alterations in AMPAR-mEPSC frequency and decay. Though the frequency is often associated with changes in the number of functional synapses, which can be ascribed to pre- or postsynaptic alterations, the decay time is linked to AMPA receptor composition; GluA2-containing AMPARs are faster, whereas GluA2-lacking AMPARs are slower [56, 57]. The altered receptor composition was confirmed by the reduction of AMPA amplitude following NASPM application. Our results support a redistribution of GluA2-lacking/GluA2containing AMPARs in adult synapses of the cortex, which could be explained by the lack of a developmental switch between AMPAR subunits.

GluA2-AMPAR subunit switching correlates with synaptic network establishment through precise dendritic spine morphological reorganisation of excitatory neurons $[41,42]$. One of the most prominent neuronal phenotypes observed in many neurodevelopmental disorders is the alteration in dendritic spine development-involving shape and/or number-suggesting that dendritic spines may serve as a common substrate for disorders that include deficits in cognition and information processing [44, 58]. The discrimination of where and when spine alterations occur in disease progression by analysing the time course of spine defects and their relationships with other endophenotypes in animal models may permit the identification of new therapeutic intervention opportunities. Here, we validated the persistence of spine aberrations in a Rab39b mouse model of ID in which RAB39B did not impair general spine development but specifically affected dendritic spine pruning and refinement. This "immaturity" phenomenon is widespread among different brain regions, as it has been shown that both the cortex and hippocampus are affected in the same direction by the lack of RAB39B.

Molecular networks controlling spines have been extensively studied; however, all the frameworks will be useful for understanding how a large number of rare genetic mutations can interrelate to disrupt spine morphology, synaptic function and neuronal circuit organisation, ultimately affecting behavioural performance in a diseasespecific manner [59].

We demonstrated the direct cause-and-effect relationship between the increase in $\mathrm{Ca}^{2+}$-permeable AMPARs due to the absence of RAB39B and the phenotypic ID-like characteristic of spine morphogenesis alteration. Until now, studies have purely associated the negative effect of increased $\mathrm{Ca}^{2+}$-permeable AMPA receptors on dendritic spine morphogenesis [43, 60-62], and others have reported alterations in AMPAR surface expression in several animal models of ID [63-65] and in post-mortem brains from human patients [66].

Rescue experiments re-expressing RAB39B or GluA2 revealed that RAB39B trafficking is necessary and sufficient for spine elimination and remodelling towards a mature spine state. Moreover, modulating $\mathrm{Ca}^{2+}$ influx with NASPM [48] as well as re-expressing RAB39B, we were able to restore the high dynamic turnover of $R a b 39 b \mathrm{KO}$ spines to WT conditions. This indicates that the absence of RAB39B and the final amount of increased $\mathrm{Ca}^{2+}$-permeable AMPARs on synapses cause spine instability, in agreement 
with the described contribution of abnormal expression of GluA2-lacking $\mathrm{Ca}^{2+}$-permeable AMPARs in neurodevelopmental disorders [43, 67, 68]. Moreover, our result on the efficacy of NASPM on $\mathrm{Ca}^{2+}$-permeable AMPARs in recovering spine abnormalities assumes therapeutic value. NASPM is already under study in murine models for neurodevelopmental disorders [64, 68], even if it is not useful for human patients due to its inability to overpass the blood brain barrier.

Mechanistically, even if we did not detect any modifications in the total amount of proteins involved in the direct modulation of spine formation, maturation and stabilisation, we cannot exclude the involvement of $\mathrm{Ca}^{2+}$ dependent signalling pathways [69, 70]. Live imaging studies on protein trafficking and intracellular neuronal localisation should identify which proteins involved in spine maturation-related pathways are specifically affected by the lack of RAB39B.

We finally analysed the explorative and cognitive performance of Rab39b KO male mice to identify reminiscent ID patient phenotypes and to evaluate the impact of immature states of dendritic spines on behavioural performance. We found that Rab39b KO mice had increased locomotor activity in the emergence test that was not observed in the novelty test, possibly due to the presence of an object in a yet-known arena, as shown by the increased curiosity towards a novel object. This is in agreement with what was described in a similar Rab39b-null mouse [38] and contrary to what was observed in different genetically GluA2-modified mice without GluA2 subunits [71].

Concerning cognitive functions, Rab39b KO mice showed a slight deficit in the radial maze working memory task, which was also reported by Niu et al. with different learning paradigms [39] and a drastic impairment in trace fear conditioning.

Fear conditioning acquisition is characterised by a transient increase in neuronal surface expression of $\mathrm{Ca}^{2+}$ permeable AMPARs in the lateral amygdala and in the hippocampus; these AMPARs are subsequently converted in $\mathrm{Ca}^{2+}$-impermeable AMPARs for memory consolidation [72]. In line with this observation, we suggest that the cognitive deficit observed in Rab39b KO mice is caused by the complete absence of GluA2-AMPAR trafficking to the neuronal surface that hinders a $\mathrm{Ca}^{2+}$-permeable frozen AMPAR scenario.

Two recent studies [38, 39] reported the generation of Rab39b KO mice by using different techniques. Zang et al. [38] reported that Rab39b KO mice had cortical neurogenesis deficits, macrocephaly, and social and motor deficits. Similar behavioural performance was observed by Niu et al. [39], demonstrating short-term working memory deficits, but the authors did not observe macrocephaly in agreement with our results. As suggested [39], this discrepancy may be caused by defects in metabolic or different biological processes between different genetic backgrounds (C57B1/6J vs $\mathrm{C} 57 \mathrm{~B} 1 / 6 \mathrm{~N})$ or by the mouse age analysed. Moreover, both studies identified abnormal activation of the PI3K-AKT-mTOR pathway, which is important in regulating cell differentiation, proliferation and metabolism during development. As we described previously, even if we did not detect any modifications in the total amount of proteins analysed, additional experiments that analyse this pathway in our model will be useful.

In summary, our study demonstrated the importance of RAB39B-mediated AMPAR trafficking in spine remodelling and in cognition and may contribute to explaining the aetiology of RAB39B-related ID.

Acknowledgements We thank the core facilities established by IRCCS Ospedale San Raffaele and Università Vita-Salute San Raffaele: ALEMBIC for the microscopy technical support, in particular in the persons of Dr Valeria Berno and Dr Eugenia Cammarota; Preclinical Imaging Facility of EIC for MRI scanning and analysis, in particular in the persons of Prof Antonio Esposito and Dr Tamara Canu; CFCM in the person of Dr Lorenza Ronfani for the generation of Rab39b KO mouse model. This work was supported by Jerome Lejeune Foundation, France (1441-DP2015B and 1744-DP2018A) and by Italian Ministry of Health (Ricerca Finalizzata RF-201302355326).

Author contributions MLM performed biochemical and cell biology experiments and GR and SG helped MLM. GR and SG performed behavioural tests and PD analysed the data. SM, PE and CB performed electrophysiological experiments. PM performed MRI analysis. SB, VM and RMM performed the FDG experiments. MLM and PD wrote the paper. PD supervised the project.

\section{Compliance with ethical standards}

Conflict of interest The authors declare no competing interests.

Publisher's note Springer Nature remains neutral with regard to jurisdictional claims in published maps and institutional affiliations.

Open Access This article is licensed under a Creative Commons Attribution 4.0 International License, which permits use, sharing, adaptation, distribution and reproduction in any medium or format, as long as you give appropriate credit to the original author(s) and the source, provide a link to the Creative Commons license, and indicate if changes were made. The images or other third party material in this article are included in the article's Creative Commons license, unless indicated otherwise in a credit line to the material. If material is not included in the article's Creative Commons license and your intended use is not permitted by statutory regulation or exceeds the permitted use, you will need to obtain permission directly from the copyright holder. To view a copy of this license, visit http://creativecommons. org/licenses/by/4.0/.

\section{References}

1. Giannandrea M, Bianchi V, Mignogna ML, Sirri A, Carrabino S, D'Elia E, et al. Mutations in the small GTPase gene RAB39B are responsible for $\mathrm{X}$-linked mental retardation associated with 
autism, epilepsy, and macrocephaly. Am J Hum Genet. 2010;86: 185-95.

2. Andersen EF, Baldwin EE, Ellingwood S, Smith R, Lamb AN. Xq28 duplication overlapping the int22h-1/int $22 \mathrm{~h}-2$ region and including RAB39B and CLIC2 in a family with intellectual and developmental disability. Am J Med Genet A. 2014;164A:1795-801.

3. Vanmarsenille L, Giannandrea M, Fieremans N, Verbeeck J, Belet S, Raynaud M, et al. Increased dosage of RAB39B affects neuronal development and could explain the cognitive impairment in male patients with distal Xq28 copy number gains. Hum Mutat. 2014;35:377-83.

4. Woodbury-Smith M, Deneault E, Yuen RKC, Walker S, Zarrei M, Pellecchia G, et al. Mutations in RAB39B in individuals with intellectual disability, autism spectrum disorder, and macrocephaly. Mol Autism. 2017;8:59.

5. Wilson GR, Sim JC, McLean C, Giannandrea M, Galea CA, Riseley JR, et al. Mutations in RAB39B cause X-linked intellectual disability and early-onset Parkinson disease with alphasynuclein pathology. Am J Hum Genet. 2014;95:729-35.

6. Lesage S, Bras J, Cormier-Dequaire F, Condroyer C, Nicolas A, Darwent L, et al. Loss-of-function mutations in RAB39B are associated with typical early-onset Parkinson disease. Neurol Genet. 2015;1:e9.

7. Mata IF, Jang Y, Kim CH, Hanna DS, Dorschner MO, Samii A, et al. The RAB39B p.G192R mutation causes X-linked dominant Parkinson's disease. Mol Neurodegener. 2015;10:50.

8. Guldner M, Schulte C, Hauser AK, Gasser T, Brockmann K. Broad clinical phenotype in Parkinsonism associated with a base pair deletion in RAB39B and additional POLG variant. Parkinsonism Relat Disord. 2016;31:148-50.

9. Hodges K, Brewer SS, Labbe C, Soto-Ortolaza AI, Walton RL, Strongosky AJ, et al. RAB39B gene mutations are not a common cause of Parkinson's disease or dementia with Lewy bodies. Neurobiol Aging. 2016;45:107-8.

10. Shi CH, Zhang SY, Yang ZH, Yang J, Shang DD, Mao CY, et al. A novel RAB39B gene mutation in X-linked juvenile parkinsonism with basal ganglia calcification. Mov Disord. 2016;31: 1905-9.

11. Ciammola A, Carrera P, Di Fonzo A, Sassone J, Villa R, Poletti B, et al. X-linked Parkinsonism with Intellectual Disability caused by novel mutations and somatic mosaicism in RAB39B gene. Parkinsonism Relat Disord. 2017;44:142-6.

12. Zerial M, McBride $H$. Rab proteins as membrane organizers. Nat Rev Mol Cell Biol. 2001;2:107-17.

13. Mignogna ML, Giannandrea M, Gurgone A, Fanelli F, Raimondi F, Mapelli L, et al. The intellectual disability protein RAB39B selectively regulates GluA2 trafficking to determine synaptic AMPAR composition. Nat Commun. 2015;6:6504.

14. Isaac JT, Ashby MC, McBain CJ. The role of the GluR2 subunit in AMPA receptor function and synaptic plasticity. Neuron. 2007;54:859-71.

15. Liu SJ, Zukin RS. Ca2+-permeable AMPA receptors in synaptic plasticity and neuronal death. Trends Neurosci. 2007;30:126-34.

16. Cantanelli P, Sperduti S, Ciavardelli D, Stuppia L, Gatta V, Sensi SL. Age-Dependent Modifications of AMPA Receptor Subunit Expression Levels and Related Cognitive Effects in 3xTg-AD Mice. Front Aging Neurosci. 2014;6:200.

17. Bourgeron T. From the genetic architecture to synaptic plasticity in autism spectrum disorder. Nat Rev Neurosci. 2015;16:551-63.

18. Neri G, Schwartz CE, Lubs HA, Stevenson RE. X-linked intellectual disability update 2017. Am J Med Genet A. 2018;176: 1375-88.

19. Fiala JC, Feinberg M, Popov V, Harris KM. Synaptogenesis via dendritic filopodia in developing hippocampal area CA1. J Neurosci. 1998;18:8900-11.
20. Sorra KE, Fiala JC, Harris KM. Critical assessment of the involvement of perforations, spinules, and spine branching in hippocampal synapse formation. J Comp Neurol. 1998;398: 225-40.

21. Lendvai B, Stern EA, Chen B, Svoboda K. Experience-dependent plasticity of dendritic spines in the developing rat barrel cortex in vivo. Nature. 2000;404:876-81.

22. Zuo Y, Lin A, Chang P, Gan WB. Development of long-term dendritic spine stability in diverse regions of cerebral cortex. Neuron. 2005;46:181-9.

23. Mostany R, Anstey JE, Crump KL, Maco B, Knott G, PorteraCailliau C. Altered synaptic dynamics during normal brain aging. J Neurosci. 2013;33:4094-104.

24. Jiang W, Bikard D, Cox D, Zhang F, Marraffini LA. RNA-guided editing of bacterial genomes using CRISPR-Cas systems. Nat Biotechnol. 2013;31:233-9.

25. Stemmer M, Thumberger T, Del Sol Keyer M, Wittbrodt J, Mateo JL. CCTop: an Intuitive, Flexible and Reliable CRISPR/Cas9 Target Prediction Tool. PLoS ONE. 2015;10:e0124633.

26. Gompers AL, Su-Feher L, Ellegood J, Copping NA, Riyadh MA, Stradleigh TW, et al. Germline Chd8 haploinsufficiency alters brain development in mouse. Nat Neurosci. 2017;20: 1062-73.

27. Pedrotti S, Caccia R, Neguembor MV, Garcia-Manteiga JM, Ferri G, de Palma C, et al. The Suv420h histone methyltransferases regulate PPAR-gamma and energy expenditure in response to environmental stimuli. Sci Adv. 2019;5:eaav1472.

28. Seibenhener ML, Wooten MW. Isolation and culture of hippocampal neurons from prenatal mice. J Vis Exp. 2012;65:3634.

29. Benavides-Piccione R, Ballesteros-Yanez I, DeFelipe J, Yuste R. Cortical area and species differences in dendritic spine morphology. J Neurocytol. 2002;31:337-46.

30. Michaluk P, Wawrzyniak M, Alot P, Szczot M, Wyrembek P, Mercik K, et al. Influence of matrix metalloproteinase MMP-9 on dendritic spine morphology. J Cell Sci. 2011;124:3369-80.

31. Lin YC, Yeckel MF, Koleske AJ. Abl2/Arg controls dendritic spine and dendrite arbor stability via distinct cytoskeletal control pathways. J Neurosci. 2013;33:1846-57.

32. Bellone C, Nicoll RA. Rapid bidirectional switching of synaptic NMDA receptors. Neuron. 2007;55:779-85.

33. Madani R, Kozlov S, Akhmedov A, Cinelli P, Kinter J, Lipp HP, et al. Impaired explorative behavior and neophobia in genetically modified mice lacking or overexpressing the extracellular serine protease inhibitor neuroserpin. Mol Cell Neurosci. 2003;23: 473-94.

34. Wolfer DP, Lipp HP. A new computer program for detailed offline analysis of swimming navigation in the Morris water maze. $\mathrm{J}$ Neurosci Methods. 1992;41:65-74.

35. Bianchi V, Gambino F, Muzio L, Toniolo D, Humeau Y, D'Adamo P. Forebrain deletion of alphaGDI in adult mice worsens the pre-synaptic deficit at cortico-lateral amygdala synaptic connections. PLoS ONE. 2012;7:e29763.

36. D'Adamo P, Masetti M, Bianchi V, More L, Mignogna ML, Giannandrea $\mathrm{M}$, et al. RAB GTPases and RAB-interacting proteins and their role in the control of cognitive functions. Neurosci Biobehav Rev. 2014;46:302-14.

37. D'Adamo P, Welzl H, Papadimitriou S, Raffaele di Barletta M, Tiveron C, Tatangelo L, et al. Deletion of the mental retardation gene Gdi1 impairs associative memory and alters social behavior in mice. Hum Mol Genet. 2002;11:2567-80.

38. Zhang W, Ma L, Yang M, Shao Q, Xu J, Lu Z, et al. Cerebral organoid and mouse models reveal a RAB39b-PI3K-mTOR pathway-dependent dysregulation of cortical development leading to macrocephaly/autism phenotypes. Genes Dev. 2020;34: 580-97. 
39. Niu M, Zheng N, Wang Z, Gao Y, Luo X, Chen Z, et al. RAB39B Deficiency Impairs Learning and Memory Partially Through Compromising Autophagy. Front Cell Dev Biol. 2020;8:598622.

40. Greger IH, Khatri L, Ziff EB. RNA editing at arg607 controls AMPA receptor exit from the endoplasmic reticulum. Neuron. 2002;34:759-72.

41. Liao D, Zhang X, O’Brien R, Ehlers MD, Huganir RL. Regulation of morphological postsynaptic silent synapses in developing hippocampal neurons. Nat Neurosci. 1999;2:37-43.

42. Petralia RS, Esteban JA, Wang YX, Partridge JG, Zhao HM, Wenthold RJ, et al. Selective acquisition of AMPA receptors over postnatal development suggests a molecular basis for silent synapses. Nat Neurosci. 1999;2:31-6.

43. Salpietro V, Dixon CL, Guo H, Bello OD, Vandrovcova J, Efthymiou S, et al. AMPA receptor GluA2 subunit defects are a cause of neurodevelopmental disorders. Nat Commun. 2019;10: 3094.

44. Martinez-Cerdeno V. Dendrite and spine modifications in autism and related neurodevelopmental disorders in patients and animal models. Dev Neurobiol. 2017;77:393-404.

45. Paolicelli RC, Bolasco G, Pagani F, Maggi L, Scianni M, Panzanelli $\mathrm{P}$, et al. Synaptic pruning by microglia is necessary for normal brain development. Science. 2011;333:1456-8.

46. Chen CC, Lu J, Zuo Y. Spatiotemporal dynamics of dendritic spines in the living brain. Front Neuroanat. 2014;8:28.

47. Baj G, Patrizio A, Montalbano A, Sciancalepore M, Tongiorgi E. Developmental and maintenance defects in Rett syndrome neurons identified by a new mouse staging system in vitro. Front Cell Neurosci. 2014;8:18.

48. Bariselli S, Hornberg H, Prevost-Solie C, Musardo S, HatstattBurkle L, Scheiffele P, et al. Role of VTA dopamine neurons and neuroligin 3 in sociability traits related to nonfamiliar conspecific interaction. Nat Commun. 2018;9:3173.

49. MacInnis BL, Campenot RB. Regulation of Wallerian degeneration and nerve growth factor withdrawal-induced pruning of axons of sympathetic neurons by the proteasome and the MEK/Erk pathway. Mol Cell Neurosci. 2005;28:430-9.

50. Pi HJ, Otmakhov N, El Gaamouch F, Lemelin D, De Koninck P, Lisman J. CaMKII control of spine size and synaptic strength: role of phosphorylation states and nonenzymatic action. Proc Natl Acad Sci USA. 2010;107:14437-42.

51. Kierdorf K, Prinz M. Factors regulating microglia activation. Front Cell Neurosci. 2013;7:44.

52. Tang G, Gudsnuk K, Kuo SH, Cotrina ML, Rosoklija G, Sosunov A, et al. Loss of mTOR-dependent macroautophagy causes autistic-like synaptic pruning deficits. Neuron. 2014;83:1131-43.

53. Bian WJ, Miao WY, He SJ, Qiu Z, Yu X. Coordinated Spine Pruning and Maturation Mediated by Inter-Spine Competition for Cadherin/Catenin Complexes. Cell. 2015;162:808-22.

54. Kumar SS, Bacci A, Kharazia V, Huguenard JR. A developmental switch of AMPA receptor subunits in neocortical pyramidal neurons. J Neurosci. 2002;22:3005-15.

55. Brill J, Huguenard JR. Sequential changes in AMPA receptor targeting in the developing neocortical excitatory circuit. J Neurosci. 2008;28:13918-28.

56. Jonas P. The Time Course of Signaling at Central Glutamatergic Synapses. N Physiol Sci. 2000;15:83-9.
57. Lu W, Shi Y, Jackson AC, Bjorgan K, During MJ, Sprengel R, et al. Subunit composition of synaptic AMPA receptors revealed by a single-cell genetic approach. Neuron. 2009;62:254-68.

58. Kaufmann WE, Moser HW. Dendritic anomalies in disorders associated with mental retardation. Cereb Cortex. 2000;10: 981-91.

59. Penzes P, Cahill ME, Jones KA, VanLeeuwen JE, Woolfrey KM. Dendritic spine pathology in neuropsychiatric disorders. Nat Neurosci. 2011;14:285-93.

60. Jia Z, Agopyan N, Miu P, Xiong Z, Henderson J, Gerlai R, et al. Enhanced LTP in mice deficient in the AMPA receptor GluR2. Neuron. 1996;17:945-56.

61. Sans N, Vissel B, Petralia RS, Wang YX, Chang K, Royle GA, et al. Aberrant formation of glutamate receptor complexes in hippocampal neurons of mice lacking the GluR2 AMPA receptor subunit. J Neurosci. 2003;23:9367-73.

62. Tanaka M, Senda T, Hirashima N. Expression of the GluA2 subunit of glutamate receptors is required for the normal dendritic differentiation of cerebellar Purkinje cells. Neurosci Lett. 2017;657:22-26.

63. Calfa G, Li W, Rutherford JM, Pozzo-Miller L. Excitation/inhibition imbalance and impaired synaptic inhibition in hippocampal area CA3 of Mecp2 knockout mice. Hippocampus. 2015;25: $159-68$.

64. Li W, Xu X, Pozzo-Miller L. Excitatory synapses are stronger in the hippocampus of Rett syndrome mice due to altered synaptic trafficking of AMPA-type glutamate receptors. Proc Natl Acad Sci USA. 2016;113:E1575-1584.

65. Murru L, Vezzoli E, Longatti A, Ponzoni L, Falqui A, Folci A, et al. Pharmacological Modulation of AMPAR Rescues Intellectual Disability-Like Phenotype in Tm4sf2-/y Mice. Cereb Cortex. 2017;27:5369-84.

66. Purcell AE, Jeon OH, Zimmerman AW, Blue ME, Pevsner J. Postmortem brain abnormalities of the glutamate neurotransmitter system in autism. Neurology. 2001;57:1618-28.

67. Hackmann K, Matko S, Gerlach EM, von der Hagen M, Klink B, Schrock E, et al. Partial deletion of GLRB and GRIA2 in a patient with intellectual disability. Eur J Hum Genet. 2013;21:112-4.

68. Yennawar M, White RS, Jensen FE. AMPA Receptor Dysregulation and Therapeutic Interventions in a Mouse Model of CDKL5 Deficiency Disorder. J Neurosci. 2019;39:4814-28.

69. Kumar V, Zhang MX, Swank MW, Kunz J, Wu GY. Regulation of dendritic morphogenesis by Ras-PI3K-Akt-mTOR and RasMAPK signaling pathways. J Neurosci. 2005;25:11288-99.

70. Joensuu M, Lanoue V, Hotulainen P. Dendritic spine actin cytoskeleton in autism spectrum disorder. Prog Neuropsychopharmacol Biol Psychiatry. 2018;84:362-81.

71. Konen LM, Wright AL, Royle GA, Morris GP, Lau BK, Seow $\mathrm{PW}$, et al. A new mouse line with reduced GluA2 Q/R site RNA editing exhibits loss of dendritic spines, hippocampal CA1neuron loss, learning and memory impairments and NMDA receptor-independent seizure vulnerability. Mol Brain. 2020; 13:27.

72. Torquatto KI, Menegolla AP, Popik B, Casagrande MA, de Oliveira Alvares L. Role of calcium-permeable AMPA receptors in memory consolidation, retrieval and updating. Neuropharmacology. 2019;144:312-8. 